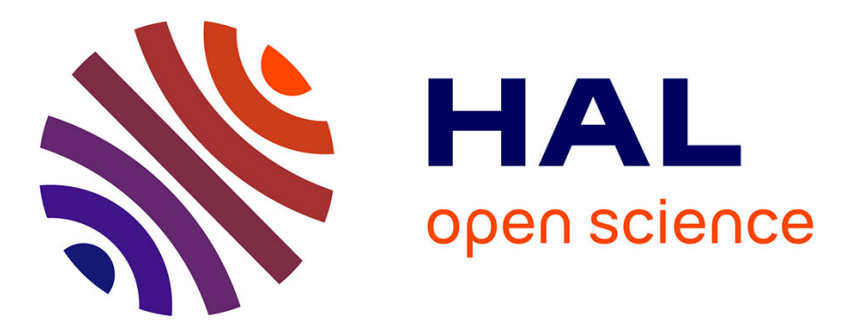

\title{
A simple phase transition relaxation solver for liquid-vapor flows
}

Alexandre Chiapolino, Pierre Boivin, Richard Saurel

\section{To cite this version:}

Alexandre Chiapolino, Pierre Boivin, Richard Saurel. A simple phase transition relaxation solver for liquid-vapor flows. International Journal for Numerical Methods in Fluids, 2017, 83 (7), pp.583-605. 10.1002/fld.4282 . hal-01359203

\section{HAL Id: hal-01359203 https://hal.science/hal-01359203}

Submitted on 2 Sep 2016

HAL is a multi-disciplinary open access archive for the deposit and dissemination of scientific research documents, whether they are published or not. The documents may come from teaching and research institutions in France or abroad, or from public or private research centers.
L'archive ouverte pluridisciplinaire HAL, est destinée au dépôt et à la diffusion de documents scientifiques de niveau recherche, publiés ou non, émanant des établissements d'enseignement et de recherche français ou étrangers, des laboratoires publics ou privés. 


\title{
A simple phase transition relaxation solver for liquid-vapor flows
}

\author{
Alexandre Chiapolino ${ }^{\mathrm{a}, \mathrm{b}}$, Pierre Boivin ${ }^{1 \mathrm{a}}$, Richard Saurel ${ }^{\mathrm{a}, \mathrm{b}, \mathrm{c}}$ \\ ${ }^{a}$ Aix Marseille Univ, CNRS, Centrale Marseille, M2P2, Marseille, France \\ ${ }^{b}$ RS2N, Chemin de Gaumin, 83640 Saint-Zacharie, France \\ ${ }^{c}$ University Institute of France
}

\begin{abstract}
Determining liquid-vapor phase equilibrium is often required in multiphase flow computations. Existing equilibrium solvers are either accurate but computationally expensive, or cheap but inaccurate. The present paper aims at building a fast and accurate specific phase equilibrium solver, specifically devoted to unsteady multiphase flow computations. Moreover, the solver is efficient at phase diagram bounds, where non-equilibrium pure liquid and pure gas are present. It is systematically validated against solutions based on an accurate (but expensive) solver. Its capability to deal with cavitating, evaporating and condensing two-phase flows is highlighted on severe test problems both $1 \mathrm{D}$ and $2 \mathrm{D}$.
\end{abstract}

Keywords: multiphase flows, interfaces, hyperbolic systems, relaxation, phase transition.

\section{Introduction}

Most multiphase flow computations face phase transition modelling and one of the difficulties is to adopt the correct mass transfer model, appropriate to a given situation. Some approaches deal with mixtures out of thermal and velocity equilibrium. When it is possible to determine the specific interfacial area separating the liquid and gas phases, determination of the mass transfer rate may be done on the basis of Nusselt and Sherwood correlations. Such a method was derived for spray evaporation by Ambramzon and Sirignano (1989) [1] and atmospheric flows by Jacobson (2005) [2]. Generalization to flashing and condensing sprays was done in Furfaro and Saurel (2016) []].

\footnotetext{
${ }^{1}$ Corresponding author: pierre.boivin@univ-amu.fr
} 
However, determination of the specific interfacial area in two-phase mixtures is possible only for droplets and bubbly flows. When the topology is arbitrary, only limit case computations are possible, assuming the absence of mass transfer if the interfacial area is supposed to be very small, or assuming infinitely fast mass transfer (local thermodynamic equilibrium) if the interfacial area is supposed to be very large. When such an assumption is made, an appropriate equilibrium solver is needed.

The present paper deals with the building of such an equilibrium solver when non-equilibrium hyperbolic models, such as Baer and Nunziato's (1986) [4] are considered. However, the present method is not restricted to such a model, but is also valid for its reduced versions such as the 5-equation model of Kapila et al. (2001) [5] and its extension for cavitating flows, Saurel et al. (2008) [6], Le Martelot et al. (2013) [7]. The Homogeneous Relaxation Model (HRM) and Homogeneous Equilibrium Model (HEM) (Downar-Zapolski et al. (1996) [8], Barret et al. (2002) [9]) being also reduced versions of these models with respectively 4 and 3 equations, the present phase transition solver similarly applies to them.

The theoretical link between these models was derived on the basis of asymptotic analysis in Saurel et al. (2008) [6], and more systematically by Lund (2012) [10]. Basically the present phase transition solver may be used each time both liquid and gas compressibility are considered, as all formerly cited models consider this effect, and this effect is responsible for their hyperbolic nature.

The 5-4-3-equation models are able to consider mixtures of fluids evolving respectively in mechanical, mechanical and thermal, and thermodynamic equilibrium. As they involve a single velocity (velocity disequilibrium is indeed absent), they are restricted mainly to specific applications such as:

- Cavitating flows, as it appears impossible in practice to address specific interfacial area determination and consequently model velocity slip. Computational examples of such flows are given for instance in Singhal et al. (2002) [11], Petitpas et al. (2009) [12], Le Martelot et al. (2013) [7] and Saurel et al. (2016) [13].

- Flashing and condensing flows, as they are high-speed flows and subject to stiff thermo- 
dynamic relaxation.

- Interfacial flows, as the same equations deal with the direct numerical simulation of boiling flows at sub-bubble scale (Le Martelot et al. (2014) [14], Saurel et al. (2016) [13]).

Therefore the equilibrium solver addressed in the present work is a key point of the 7-5-4-3equation hyperbolic two phase flow models as it computes local thermodynamic equilibrium, this feature being important in many situations. The building of such an equilibrium solver has been addressed in Orbey et al. (1998) [15], Allaire et al. (2007) [16], Faccanoni et al. (2012) [17] and Le Metayer et al. (2013) [18] on the basis of a highly non-linear-algebraic model made from the saturation conditions, mixture mass and mixture energy definitions. This system may cause difficulties as a result of non-linearities and single phase bounds of pure liquid and pure vapor, where it becomes ill posed.

In the present paper a novel approach is promoted where the solution relaxes weakly (smoothly during time evolution) to the correct solution, on the basis of some estimates. After providing the background and context of the model in Sections 1 and 2 2 , the two main ideas constituting the present model are successively detailed in Sections 3 and 4 :

- Limitation of the relaxation term, following a Minmod-type procedure, reminiscent of slope limiters in high-order hyperbolic solvers (Van Leer et al. (1979) [19]).

- Approximate analytical estimation of the pressure at thermodynamic equilibrium.

These two treatments lead to a faster procedure than the usual iterative process: reported computation times can be halved with the new algorithm. In addition, the algorithm described hereafter presents a very simple implementation, which is also a significant improvement over iterative procedures. Indeed, the method properly deals with limit cases, when only liquid or vapor is present, free to evolve out of the saturation conditions. The last Sections (5, 6 and 7) are dedicated to illustrating the capabilities of the method, with several computational 1D and 2D examples of flows with evaporation, condensation, cavitation in the bulk fluid and at liquid-vapor interfaces. 


\section{Flow model}

The phase transition relaxation solver may be used with models mentioned previously (with 7, 5, 4 and 3 partial differential equations) but its presentation is simplified in the context of the 4-equation model (often called HRM) as the solver directly connects the 4 and 3-equation models. When dealing with more sophisticated formulations, for instance the 7-equation model, extra ingredients have to be presented, such as velocity and pressure relaxation solvers (see for example [20]). Here, there is a single step that makes the connection from the 4-equation model (modeling mixtures out of thermodynamic equilibrium) and the 3-equation model (mixtures in full equilibrium). Therefore, for the sake of simplicity the 4-equation model (HRM) is considered in the present paper as starting point. The corresponding flow model reads,

$$
\begin{cases}\frac{\partial \rho}{\partial t}+\operatorname{div}(\rho \mathbf{u}) & =0, \\ \frac{\partial \rho \mathbf{u}}{\partial t}+\operatorname{div}(\rho \mathbf{u} \otimes \mathbf{u}+p \underline{\underline{I}}) & =0, \\ \frac{\partial \rho E}{\partial t}+\operatorname{div}((\rho E+p) \mathbf{u}) & =0, \\ \frac{\partial \rho Y_{l}}{\partial t}+\operatorname{div}\left(\rho Y_{l} \mathbf{u}\right) & =0,\end{cases}
$$

alternatively, the last equation can be written as,

$$
\frac{\partial \rho_{l} \alpha_{l}}{\partial t}+\operatorname{div}\left(\rho_{l} \alpha_{l} \mathbf{u}\right)=0,
$$

where $Y_{l, g}, \alpha_{l, g}, \rho_{l, g}$ denote respectively the mass fraction, the volume fraction and the material density of the liquid $(l$ subscript) and gas $(g$ subscript) phases. $\rho$ represents the mixture density, $\mathbf{u}$ represents the mixture centre of mass velocity, $p$ denotes the mixture pressure and $E$ the mixture total energy $\left(E=e+u^{2} / 2\right)$. The mixture internal energy is defined as $e=Y_{l} e_{l}+Y_{g} e_{g}$. Mass transfer has been omitted in System (1) as it is addressed later.

System (1) is clearly reminiscent of the reactive (or multicomponent) Euler equations widely used in chemically reacting flows. However, the thermodynamic closure differs significantly from the one used in gas mixtures since each phase is assumed to occupy its own volume. Indeed the 
mixture equation of state (EOS) is a consequence of the following algebraic system:

$$
\left\{\begin{array}{l}
T_{l}=T_{g}=T, \\
e=Y_{l} e_{l}(T, p)+Y_{g} e_{g}(T, p), \\
p_{l}=p_{g}=p, \\
v=Y_{l} v_{l}(T, p)+Y_{g} v_{g}(T, p),
\end{array}\right.
$$

where $v_{l}, v_{g}$ and $v$ are respectively the specific volumes of the liquid, gas, and mixture.

In this frame, both liquid and gas require their own equation of state (EOS), with parameters carefully chosen to fit the phase diagram. The building of such EOS has been addressed in Le Métayer et al. (2004) [21], on the basis of the stiffened gas (SG) EOS, an improved formulation (NASG) being available as well [22]. The main formulas for the SG EOS read for a given phase $k=l, g$,

$$
\left\{\begin{aligned}
p_{k}\left(\rho_{k}, e_{k}\right) & =\rho_{k}\left(\gamma_{k}-1\right)\left(e_{k}-q_{k}\right)-\gamma_{k} p_{\infty, k} \\
T_{k}\left(p_{k}, \rho_{k}\right) & =\frac{p_{k}+p_{\infty, k}}{\rho_{k} C_{v, k}\left(\gamma_{k}-1\right)} \\
g_{k}\left(p_{k}, T_{k}\right) & =\left(\gamma_{k} C_{v, k}-q_{k}^{\prime}\right) T_{k}-C_{v, k} T_{k} \ln \left[\frac{T_{k}^{\gamma_{k}}}{\left(p_{k}+p_{\infty, k}\right)^{\gamma_{k}-1}}\right]+q_{k} \\
c_{k}\left(p_{k}, \rho_{k}\right) & =\sqrt{\gamma_{k} \frac{p_{k}+p_{\infty, k}}{\rho_{k}}}
\end{aligned}\right.
$$

where the following parameters are needed for each phase: $\gamma_{k}, p_{\infty, k}, C_{v, k}, q_{k}$, and $q_{k}^{\prime}$. From Eqs. (3), two other equations are found,

$$
\left\{\begin{aligned}
v_{k}\left(p_{k}, T_{k}\right) & =\frac{\left(\gamma_{k}-1\right) C_{v, k} T_{k}}{p_{k}+p_{\infty, k}} \\
e_{k}\left(p_{k}, T_{k}\right) & =\frac{p_{k}+\gamma_{k} p_{\infty, k}}{p_{k}+p_{\infty, k}} C_{v, k} T_{k}+q_{k}
\end{aligned}\right.
$$

As shown in [21] there is no difficulty to obtain these parameters once the saturation curves $\left(p_{\text {sat }}(T), v_{g, s a t}(T), v_{l, s a t}(T), h_{g, s a t}(T), h_{l, s a t}(T)\right)$ are known. Saturation pressure and temperature obeying formulation (3) are linked through the saturation curve,

$$
\ln \left(p_{\text {sat }}+p_{\infty, g}\right)=A+\frac{B}{T_{\text {sat }}}+C \ln \left(T_{\text {sat }}\right)+D \ln \left(p_{\text {sat }}+p_{\infty, l}\right)
$$


with,

$$
A=\frac{C_{p, l}-C_{p, g}+q_{g}^{\prime}-q_{l}^{\prime}}{C_{p, g}-C_{v, g}}, \quad B=\frac{q_{l}-q_{g}}{C_{p, g}-C_{v, g}}, \quad C=\frac{C_{p, g}-C_{p, l}}{C_{p, g}-C_{v, g}}, \quad D=\frac{C_{p, l}-C_{v, l}}{C_{p, g}-C_{v, g}} .
$$

which is equivalent to equaling the Gibbs free energies $g_{l}$ and $g_{g}$ from the Stiffened gas EOS (3) (see [21] for details).

For liquid water and steam, the fluid parameters, optimized in the $[298-473 \mathrm{~K}]$ temperature range are given in Table 1 ,

\begin{tabular}{|l|c|c|}
\hline Coefficients & Liquid phase & Vapor phase \\
\hline$C_{p}(\mathrm{~J} / \mathrm{kg} / \mathrm{K})$ & 4267 & 1487 \\
\hline$C_{v}(\mathrm{~J} / \mathrm{kg} / \mathrm{K})$ & 1816 & 1040 \\
\hline$\gamma$ & 2.35 & 1.43 \\
\hline$P_{\infty}(\mathrm{Pa})$ & $10^{9}$ & 0 \\
\hline$q(\mathrm{~J} / \mathrm{kg})$ & $-116710^{3}$ & $203010^{3}$ \\
\hline$q^{\prime}(\mathrm{J} / \mathrm{kg} / \mathrm{K})$ & 0 & $-2310^{3}$ \\
\hline
\end{tabular}

Table 1: Stiffened gas coefficients for water determined in the temperature range [298 - $473 \mathrm{~K}]$

These parameters are used in the computational examples of the present paper. With the mixture thermodynamic closure (2), it is straightforward to derive the following analytical relations for the mixture temperature, energy and pressure, that correspond to the mixture EOS:

$$
\left\{\begin{array}{l}
T=T\left(v, p, Y_{l}\right), \\
e=e\left(T, p, Y_{l}\right), \\
p=p\left(v, e, Y_{l}\right) .
\end{array}\right.
$$

The thermodynamic closure presented in [21] or its improved formulation [22] is very convenient, as the above relations (7) are fully explicit for the mixture [13, 14]. The mixture temperature reads,

$$
T\left(v, p, Y_{l}\right)=v\left(\frac{\left(\gamma_{l}-1\right) Y_{l} C_{v, l}}{p+p_{\infty, l}}+\frac{\left(\gamma_{g}-1\right) Y_{g} C_{v, g}}{p+p_{\infty, g}}\right)^{-1}
$$


the mixture internal energy reads,

$$
e\left(T, p, Y_{l}\right)=Y_{l}\left(C_{v, l} T \frac{p+\gamma_{l} p_{\infty, l}}{p+p_{\infty, l}}+q_{l}\right)+Y_{g}\left(C_{v, g} T \frac{p+\gamma_{g} p_{\infty, g}}{p+p_{\infty, g}}+q_{g}\right),
$$

and the pressure reads,

$$
p\left(v, e, Y_{l}\right)=\frac{1}{2}\left(A_{l}+A_{g}-\left(p_{\infty, l}+p_{\infty, g}\right)\right)+\sqrt{\frac{1}{4}\left(A_{g}-A_{l}-\left(p_{\infty, g}-p_{\infty, l}\right)\right)^{2}+A_{l} A_{g}}
$$

with,

$$
A_{k}=\frac{Y_{k}\left(\gamma_{k}-1\right) C_{v, k}}{\left(Y_{l} C_{v, l}+Y_{g} C_{v, g}\right)}\left(\frac{e-\left(Y_{l} q_{l}+Y_{g} q_{g}\right)}{v}-p_{\infty, k}\right),
$$

where the subscript $k$ denotes liquid $(l)$ and gas $(g)$ phases (see [14] for details).

Albeit the apparent simplicity of the thermodynamic closure chosen, the phase transition model presented here may be extended to other thermodynamic closures given each phase EOS is convex. Such extension is immediate with NASG [22].

The reactive Euler equations govern the propagation of three waves throughout space (Fig. 1). The middle wave (traveling along $\mathrm{u}$ ) is a contact discontinuity, while the left and right waves (traveling along $u \pm c, c$ being the speed of sound) are non-linear acoustic waves and can be either shocks or rarefactions.

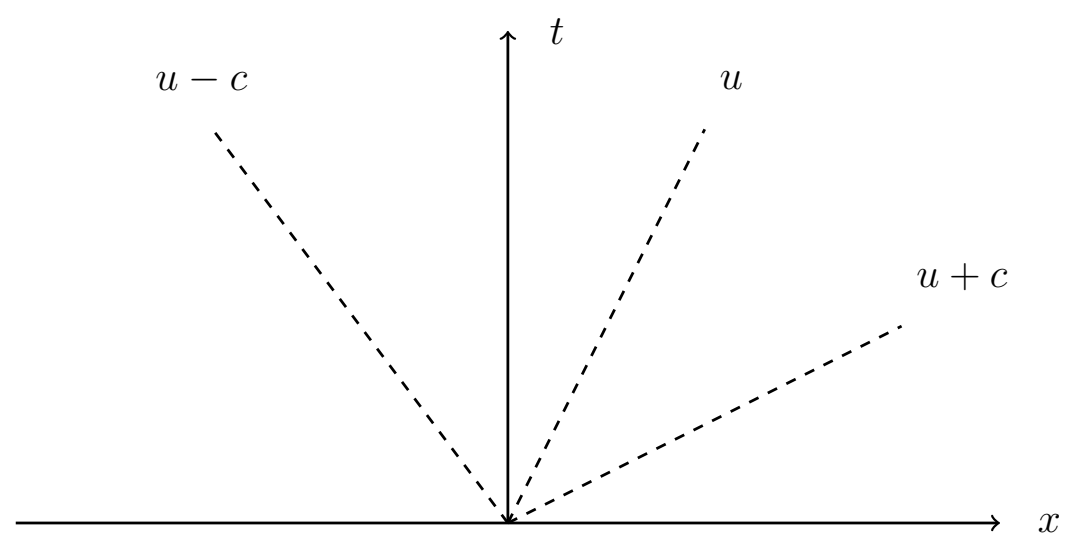

Figure 1: Schematic representation in a $(\mathrm{x}, \mathrm{t})$ diagram of the three waves present in the flow model (1).

With the thermodynamic closure (2), System (1) is hyperbolic with wave speeds $u, u+c$ and 
$u-c$. The sound speed for this system is given in Le Martelot et al. (2014) [14],

$$
\begin{aligned}
c^{2}= & \frac{1}{2}\left\{\left[e-\left(Y_{l} q_{l}+Y_{g} q_{g}\right)\right]\left(a_{1}+a_{2}\right)+\frac{\frac{1}{2}\left(\frac{\partial R_{1}}{\partial \rho}\right)_{e} R_{1}+\left(\frac{\partial R_{2}}{\partial \rho}\right)_{e}}{\sqrt{\frac{1}{4} R_{1} R_{1}+R_{2}}}\right. \\
& \left.+\frac{p}{\rho^{2}}\left(\rho\left(a_{1}+a_{2}\right)+\frac{\frac{1}{2}\left(\frac{\partial R_{1}}{\partial e}\right)_{\rho} R_{1}+\left(\frac{\partial R_{2}}{\partial e}\right)_{\rho}}{\sqrt{\frac{1}{4} R_{1} R_{1}+R_{2}}}\right)\right\}
\end{aligned}
$$

where,

$$
\left\{\begin{aligned}
& a_{1}= \frac{Y_{l}\left(\gamma_{l}-1\right) C_{v, l}}{Y_{l} C_{v, l}+Y_{g} C_{v, g}} \\
& a_{2}= \frac{Y_{g}\left(\gamma_{g}-1\right) C_{v, g}}{Y_{l} C_{v, l}+Y_{g} C_{v, g}} \\
& R_{1}= a_{2} \rho\left[e-\left(Y_{l} q_{l}+Y_{g} q_{g}\right)\right]-a_{2} p_{\infty, g}-a_{1} \rho\left[e-\left(Y_{l} q_{l}+Y_{g} q_{g}\right)\right]+a_{1} p_{\infty, l}-p_{\infty, g}+p_{\infty, l}, \\
& R_{2}=a_{1} a_{2}\left\{\rho\left[e-\left(Y_{l} q_{l}+Y_{g} q_{g}\right)\right]-p_{\infty, l}\right\}\left\{\rho\left[e-\left(Y_{l} q_{l}+Y_{g} q_{g}\right)\right]-p_{\infty, g}\right\} .
\end{aligned}\right.
$$

This sound speed can then be compared with a simpler approximation of the sound speed given by Wood [23]:

$$
\frac{1}{\rho c^{2}}=\frac{\alpha_{l}}{\rho_{l} c_{l}^{2}}+\frac{\alpha_{g}}{\rho_{g} c_{g}^{2}}
$$

As shown in Fig. 2, Wood's expression for the sound speed (Eq. (14)) is always slightly greater than the sound speed given by Eq. (12). It is thus more convenient (and simpler) for computational purposes related to the hyperbolic solver.

\section{Phase transition model}

When phase transition is addressed in System (11), the equations for the mixture mass, momentum and energy are unaffected, and only the mass fraction equation is modified through Gibbs free energy relaxation terms,

$$
\frac{\partial \rho Y_{l}}{\partial t}+\frac{\partial \rho u Y_{l}}{\partial x}=\rho \nu\left(g_{g}-g_{l}\right)
$$

where $g_{k}$ denotes the phase $\mathrm{k}$ Gibbs free energy. $\nu\left(A_{I}, T, p\right)$ represents a relaxation parameter that controls the rate at which thermodynamic equilibrium is reached. It is a function of the 

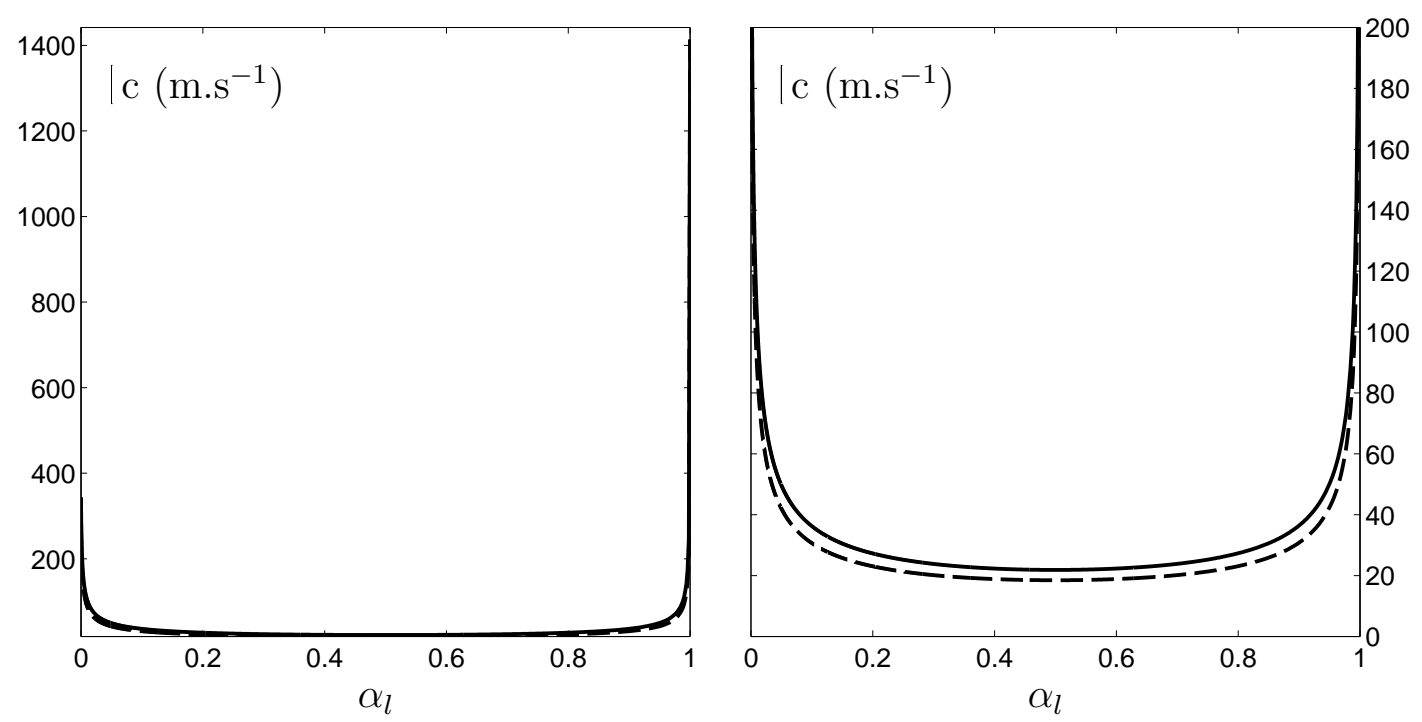

Figure 2: The speed of sound of System (11) given by Eq. (12) is compared with Wood's sound speed (Eq. (14)). Full view and close up. The thick lines represent Wood's sound speed. The dashed lines represent the augmented Euler's sound speed. The two-phase mixture is made of liquid water and air at atmospheric conditions.

interfacial area $A_{I}$, temperature and pressure. Its determination is possible only when the interfacial area $A_{I}$ is available, as with droplets and bubbly flows. In the present paper work, $\nu$ is considered very big, so that relaxation to thermodynamic equilibrium is immediate.

Using a fractional step method, phase transition is decoupled of transport and wave propagation. At each time-step of the flow solver, the following equation has to be resolved for the mass fraction $Y_{l}$,

$$
\frac{\partial\left(\rho Y_{l}\right)}{\partial t}=\frac{\rho\left(Y_{l}^{*}-Y_{l}\right)}{\tau},
$$

where $Y_{l}^{*}$ is the liquid mass fraction at thermodynamic equilibrium. This equation trivially solves to a solution exponentially tending to $Y_{l}^{*}$, with a characteristic time $\tau$. In this work, $\tau$ is assumed to be smaller than the other characteristic times of the flow model: stiff relaxation is considered. As a consequence, solving Eq. (15) at every time-step reduces to setting $Y_{l}=Y_{l}^{*}$ after each hyperbolic step.

Although the specific volume $v=1 / \rho$ and energy $e$ do not vary in the mixture during thermodynamic relaxation, the pressure and temperature do, also reaching their equilibrium 
values $\left(p^{*}, T^{*}\right)$ on the saturation curve, since $g_{l}=g_{g}$ is equivalent to the saturation condition: Eq. (5). The phase transition model thus reduces to computing the equilibrium state $\left(p^{*}, T^{*}, v, e, Y_{l}^{*}\right)$, at every time step, from the state described by $\left(p, T, v, e, Y_{l}\right)$, as represented schematically in Fig. 3.

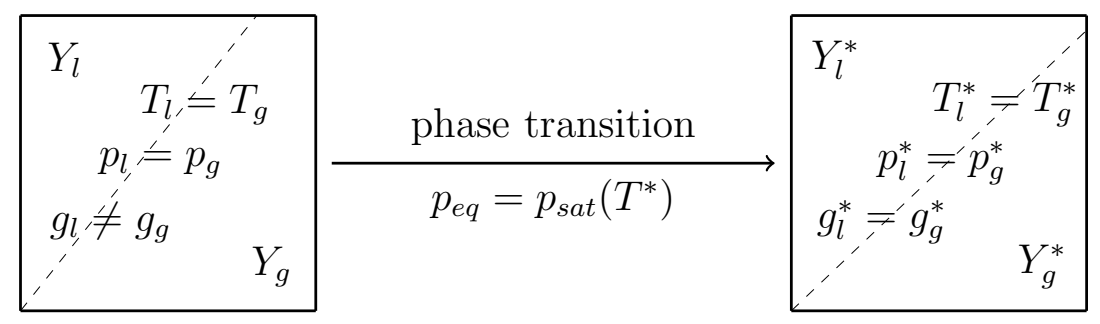

Figure 3: Representation of a control volume in the flow model during the phase transition step.

With the thermodynamic closure (2) presented above, the equilibrium state satisfies,

$$
\left\{\begin{array}{l}
p^{*}\left(v, e, Y_{l}^{*}\right)=p_{s a t}\left(T^{*}\left(v, e, Y_{l}^{*}\right)\right) \\
v=Y_{l}^{*} v_{l}\left(T^{*}, p^{*}\right)+\left(1-Y_{l}^{*}\right) v_{g}\left(T^{*}, p^{*}\right) \\
e=Y_{l}^{*} e_{l}\left(T^{*}, p^{*}\right)+\left(1-Y_{l}^{*}\right) e_{g}\left(T^{*}, p^{*}\right)
\end{array}\right.
$$

unless there is a solution in which the mixture is a pure phase (resp. $Y_{l}^{*}=0$ or $Y_{l}^{*}=1$ ), with a temperature respectively above or below the saturation temperature. The above non-linear system can be solved following an iterative algorithm such as Le Metayer et al.'s (2013) [18], summarized in Appendix, but the aim of this paper is to offer a simpler and faster alternative.

\section{Thermochemical relaxation algorithm}

Let us remind that with an iterative approach, the goal of the thermochemical relaxation is to compute accurately $Y_{l}^{*}$, the liquid mass fraction at equilibrium (or alternatively $Y_{g}^{*}$ ), while with the present method, the aim is to reach the same solution but gradually (typically 2 or 3 time steps).

The first step is to check with pure fluids existence. For numerical reasons, pure fluid conditions are considered via $\epsilon \rightarrow 0$, typically on the order of $10^{-8}$. To do this, $Y_{l}^{*}=\epsilon$ and $Y_{l}^{*}=1-\epsilon$ are successively assumed. Under these assumptions, the pressures are computed by 
use of the equation of state for the mixture (77),

$$
p=p\left(v, e, Y_{l}\right)
$$

since $v$ and $e$ are invariant through phase transition. The associated temperatures are then computed through Eq. (8),

$$
T=T\left(v, p, Y_{l}\right)
$$

The corresponding temperatures are compared to the saturation one at the current pressure $\left(T_{\text {sat }}(p)\right)$,

$$
\left\{\begin{array}{lll}
\text { if }\left(Y_{l}=\epsilon\right. & \text { and } \left.T>T_{s a t}\right) \text { then } \mathrm{Y}_{1}^{*}=\epsilon & \text { (overheated vapor) } \\
\text { if }\left(Y_{l}=1-\epsilon\right. & \text { and } \left.T<T_{\text {sat }}\right) \text { then } \mathrm{Y}_{1}^{*}=1-\epsilon & \text { (subcooled liquid) } .
\end{array}\right.
$$

If one of the two inequalities is fulfilled the equilibrium liquid mass fraction $Y_{l}^{*}$ is fully determined and no further computation is required. If none of the above statements is true, then necessarily,

$$
\epsilon<Y_{l}^{*}<1-\epsilon, \quad p^{*} \text { is unknown, }
$$

and System (17) has to be resolved. The difficulty resides in the non-trivial relationship between the saturation pressure and saturation temperature arising from Eq. (5):

$$
T_{s a t}(p)=-\frac{B}{C \cdot W\left(-\frac{B e^{A / C} p^{-1 / C}\left(p+p_{\infty, l}\right)^{D / C}}{C}\right)},
$$

where $\mathrm{W}$ is the Lambert $\mathrm{W}$ function 2 , which cannot be expressed analytically, calling for an iterative method such as Newton's.

The idea of the algorithm is to start from a rough estimate of the equilibrium pressure $p^{*}=p$, and the associated equilibrium temperature $T^{*}=T_{\text {sat }}(p)$ and reach gradually the solution. Since the pressure and temperature are related at saturation, the liquid internal energy $e_{l}(p, T)$ and specific volume $v_{l}(p, T)$ become two functions depending of $p$ only, and two values for $Y_{l}^{*}$ as

\footnotetext{
${ }^{2}$ The Lambert $\mathrm{W}$ function is defined as $z=W\left(z e^{z}\right)$.
} 
functions of the initial pressure $p$ are obtained from System (17), by either using the mixture mass definition,

$$
Y_{l}^{m}(p)=\frac{v-v_{g}(p)}{v_{l}(p)-v_{g}(p)}
$$

or the mixture internal energy definition,

$$
Y_{l}^{e}(p)=\frac{e-e_{g}(p)}{e_{l}(p)-e_{g}(p)}
$$

Indeed, these two formulas are equal only if $p$ is exactly the equilibrium pressure $p^{*}$, which is not the case a priori since the process is not isobaric.

Based on these two guesses, a strategy inspired by flux limiters used in high-order schemes [19] is adopted. Let us introduce the ratio of the liquid mass fractions variation induced by the mass $\left(Y^{m}-Y\right)$ and internal energy $\left(Y^{e}-Y\right)$ guesses for the equilibrium mass fraction,

$$
r=\frac{Y_{l}^{m}(p)-Y_{l}}{Y_{l}^{e}(p)-Y_{l}}
$$

An estimate of the equilibrium mass fraction is then obtained as,

$$
\begin{cases}\text { if } \mathrm{r}<0, & Y_{l}^{*}=Y_{l} \\ \text { if } 0<\mathrm{r}<1, & Y_{l}^{*}=Y_{l}^{\text {mass }} \\ \text { if } \mathrm{r}>1, & Y_{l}^{*}=Y_{l}^{\text {energy }} .\end{cases}
$$

Alternatively, it also expresses as,

$$
\begin{gathered}
r=\left(Y_{l}^{m}(p)-Y_{l}\right)\left(Y_{l}^{e}(p)-Y_{l}\right), \\
\begin{cases}\text { if } \mathrm{r}<0, & Y_{l}^{*}=Y_{l} \\
\text { otherwise } & Y_{l}^{*}=Y_{l}+\operatorname{sgn}\left[Y_{l}^{m}(p)-Y_{l}\right] \times \operatorname{Min}\left[\left|Y_{l}^{m}(p)-Y_{l}\right|,\left|Y_{l}^{e}(p)-Y_{l}\right|\right] .\end{cases}
\end{gathered}
$$

In the first case, the evolutions indicated by the two equilibrium guesses are discordant: one tends to evaporate whereas the other tends to condensate. The four quantities $\left(Y_{l}, Y_{l}^{*}, Y_{l}^{m}, Y_{l}^{e}\right)$ are then likely to be very close from one another, and no mass transfer is to be considered. 
In the other case, among $\left(Y_{l}^{m}, Y_{l}^{e}\right)$, the closest to the initial value $Y_{l}$ is to be chosen. In that sense, the algorithm ensures equality of Eqs. (21) and (22) in the weak sense, rather than in the strong sense. Negative mass fractions are impossible to obtain with this algorithm. This can be seen from Figs. 4, 5, 7, slopes of $Y_{g}^{e}$ and $Y_{g}^{m}$ are of different sign. Since the crossing of the two lines occurs at a positive mass fraction (which is the exact solution), only one of $Y_{g}^{e}$ and $Y_{g}^{m}$ can be negative at a time. Following the algorithm, there are then two possibilities: if the initial $Y_{g}$ (from the hyperbolic step) is between $Y_{g}^{e}$ and $Y_{g}^{m}$, then nothing happens thanks to the Minmod-like limitation. If $Y_{g}$ is not between $Y_{g}^{e}$ and $Y_{g}^{m}$, then it is necessarily above the maximum of the two (since $Y_{g}>0$ ), and the algorithm will automatically pick the closest estimation (which is then positive).

The reason for this algorithm efficiency is illustrated in Fig. 4, which presents the evolution of $Y_{l}^{m}$ and $Y_{l}^{e}$ as functions of the initial guess for the pressure, for a mixture initially away from thermodynamic equilibrium. It is seen that following the above algorithm, which in the depicted case returns $Y_{l}^{*}=Y_{l}^{e}$, gives a result within half a percent of the exact value, even though the initial state is quite far from equilibrium: $30 \mathrm{~K}$ below the saturation temperature at the initial pressure of $1 \mathrm{~atm}$.

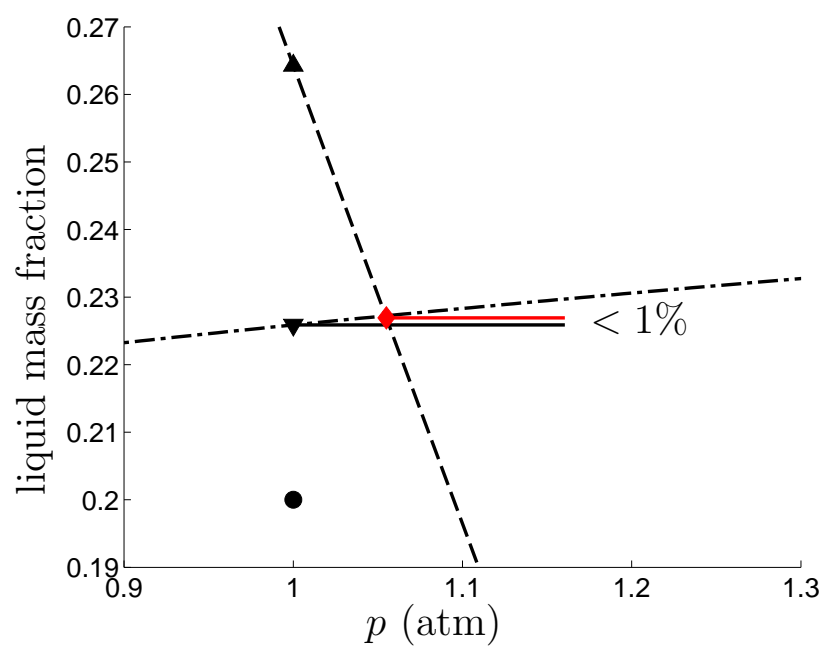

Figure 4: Evolution of $Y_{l}^{m}$ and $Y_{l}^{e}$ with $p$ (thick dashed and dashed-dotted lines resp.) corresponding to Eqs. (21) and (22). The exact values of $Y_{l}^{*}$ and $p^{*}$ are found at the crossing of the two lines $(\bullet)$. Initial state $(\bullet)$ : $p=1 \mathrm{~atm}, Y_{l}=0.2, T=T_{\text {sat }}-30 \mathrm{~K}=343 \mathrm{~K}$. The triangles represent the value of $Y_{l}^{m}(\mathbf{\Lambda})$ and $Y_{l}^{e}(\boldsymbol{\nabla})$ evaluated by the algorithm. Relative error between the exact solution $Y_{l}^{*}$ and that obtained with the algorithm is indicated. 


\section{Further improvement: Estimate of the equilibrium pressure $p^{*}$}

When the initial conditions are such that $p$ is far from the equilibrium pressure $p^{*}$, or equivalently, that a small change in $Y_{l}$ creates an important pressure variation, the above algorithm may fail. Since the liquid has a low compressibility, this will be typically the case when $Y_{l}$ is close to 1 (or, equivalently $Y_{g} \ll 1$ ). A small variation of the liquid mass fraction $Y_{l}$ when close to unity involves large variations of pressure. Figure 5. a shows such a behavior: starting from a small gas mass fraction $Y_{g}=1-Y_{l}=10^{-3}$, and far from equilibrium $\left(T=T_{\text {sat }}+30 \mathrm{~K}\right)$, the algorithm previously described would compute $Y_{g}^{m}=1-Y_{l}^{m}$ as the equilibrium mass fraction. Although the mass transfer obtained has the proper sign, which is essential to the method convergence, it is seen in Figure 5, a that $Y_{g}^{*}$ is underestimated by $77 \%$. This section aims at improving this estimate, while keeping a low computational cost.
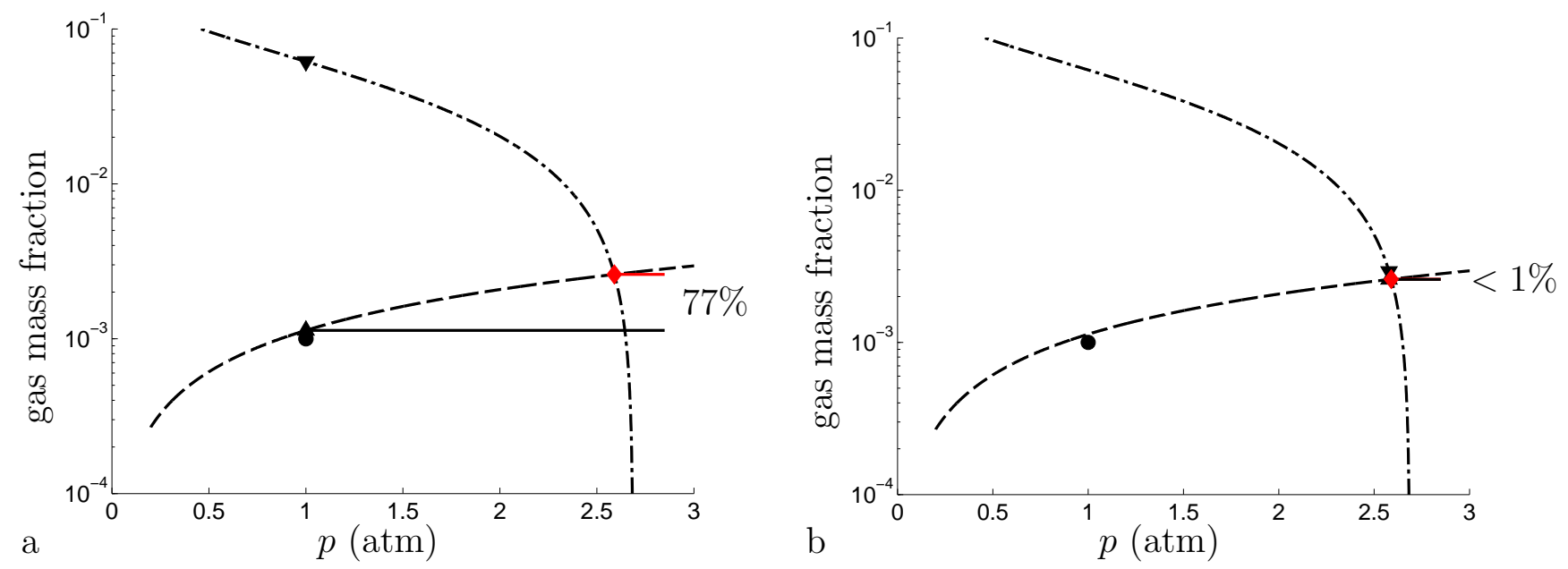

Figure 5: Evolution of $Y_{g}^{m}$ and $Y_{g}^{e}$ from Eqs. (21) and (22) near the pure liquid bound with $p$ (thick dashed and dashed-dotted lines resp). The exact values of $Y_{g}^{*}$ and $p^{*}$ are found at the crossing of the two lines $(\checkmark)$. Initial state $(\bullet): p=1 \mathrm{~atm}, Y_{g}=0.001, T=T_{\text {sat }}+30 \mathrm{~K}=403 \mathrm{~K}$. The triangles represent the value of $Y_{l}^{m}(\mathbf{\Delta})$ and $Y_{l}^{e}$ ( $\mathbf{v}$ ) evaluated by the algorithm. a) $p$ is directly used for the initial guess of $p^{*}$. b) Based on the improved guess for $p^{*}$. Relative error between the exact solution $Y_{g}^{*}$ and that obtained with the algorithm is indicated.

As it is usually the case when looking for an approximate solution, it is convenient to identify the bounds for the equilibrium pressure $p^{*}$. Let us study the variations of the equilibrium pressure $p^{*}$ as a function of an initial set of conditions $\left(p, T, v, e, Y_{g}\right)$, where $T \neq T_{\text {sat }}(p)$. The variation of the equilibrium pressure with the initial gas mass fraction $Y_{g}$ is shown in Fig. 6, for an initial pressure of $1 \mathrm{~atm}$, and a temperature $30 \mathrm{~K}$ over the corresponding saturation temperature 
$(T=403 \mathrm{~K})$. As expected, the equilibrium pressure variation is monotonic. The extrema of $p^{*}$ correspond necessarily to its value in the limits $Y_{g} \rightarrow 0$ and $Y_{g} \rightarrow 1$.

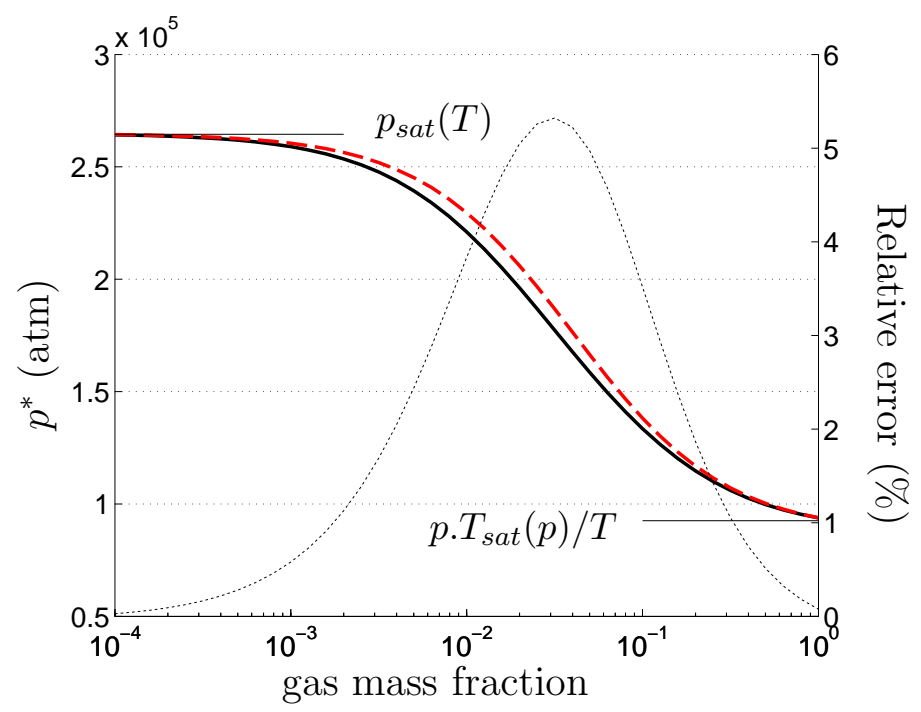

Figure 6: Evolution of $p^{*}$ as a function of an initial set of conditions $\left(p, T, v, e, Y_{g}\right)$, where $T=T_{\text {sat }}(p)+30 \mathrm{~K}$ and $p=1 \mathrm{~atm}$. Thick line: exact solution for $p^{*}$. Dashed line: approximate solution, according to Eq. (27). The two bounds for the equilibrium pressure $p^{*}$ are indicated. The dotted curve corresponds to the relative error between the exact and approximate solution, showing a maximum error close to $5 \%$.

In the limit $Y_{l} \rightarrow 1$, the mixture is mostly liquid. Mass transfer between the two phases does not modify the temperature significantly, as the energy is mainly the one of the liquid that has the highest internal heat capacity. However, a small change in $Y_{g}$ will lead to a significant pressure change. Saturation conditions lead to $T^{*}=T$ and

$$
p_{1}^{*}=p_{\text {sat }}(T)
$$

In the other limit, the mixture is mostly gas. The pressure change is then related to the temperature change as would a constant volume gas. In our case: $p / T$ is constant at constant density. The second pressure limit is then solution to $p^{*} / T_{\text {sat }}\left(p^{*}\right)=p / T$, which is approximated as

$$
p_{2}^{*}=\frac{p \cdot T_{s a t}(p)}{T} .
$$

The following step is to estimate $Y_{l}^{m}(p)$ and $Y_{l}^{e}(p)$ at the two equilibrium pressure limits $\left(p_{1}^{*}, p_{2}^{*}\right)$ defined by Eqs. (25) and (26), according to Eqs. (21) and (22). The corresponding points are shown in the Fig. 7 . 


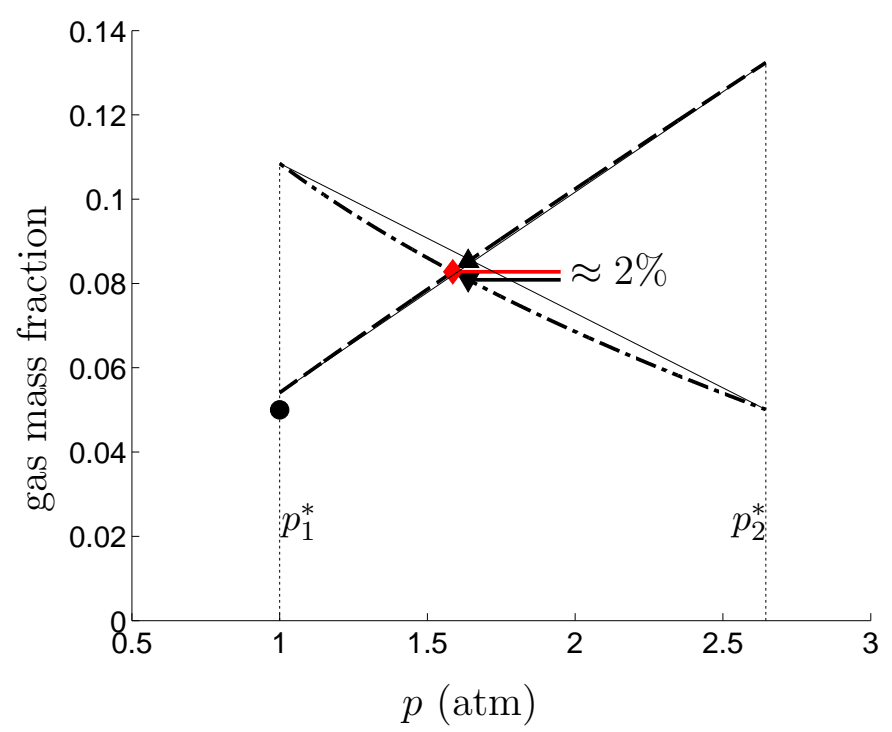

Figure 7: Evolution of $Y_{g}^{m}$ and $Y_{g}^{e}$ from Eqs. (21) and (22) with $p$ (thick dashed and dashed-dotted lines resp.). The exact values of $Y_{g}^{*}$ and $p^{*}$ are found at the crossing of the two lines $(\checkmark)$. The approximated value of $p^{*}$ is found at the crossing of the two straight lines. Initial state $(\bullet): p=1 \mathrm{~atm}, Y_{g}=0.05, T=T_{\text {sat }}+30 \mathrm{~K}=403 \mathrm{~K}$. The triangles represent the value of $Y_{l}^{m}(\mathbf{\Lambda})$ and $Y_{l}^{e}(\mathbf{v})$ evaluated by the algorithm.

Assuming $Y_{l}^{m}(p)$ and $Y_{l}^{e}(p)$ to be linear functions of $p$ between $p_{1}^{*}, p_{2}^{*}$, it is straightforward to obtain an approximate expression for the equilibrium pressure found at the intersection of $Y_{l}^{m}(p)$ and $Y_{l}^{e}(p)$,

$$
p^{*}=(1-\theta) p_{1}^{*}+\theta p_{2}^{*},
$$

where,

$$
\theta=\frac{Y_{l}^{m}\left(p_{1}^{*}\right)-Y_{l}^{e}\left(p_{1}^{*}\right)}{\left(Y_{l}^{e}\left(p_{2}^{*}\right)-Y_{l}^{m}\left(p_{2}^{*}\right)\right)-\left(Y_{l}^{e}\left(p_{1}^{*}\right)-Y_{l}^{m}\left(p_{1}^{*}\right)\right)} .
$$

Fig. 6 compares the approximate equilibrium pressure with its exact value, showing that the maximum error is of the order of $5 \%$. Note that these results are obtained for a mixture far from equilibrium initially: $T=T_{\text {sat }}(p)+30 \mathrm{~K}$. In practice, the algorithm does not have to deal with mixture that far from equilibrium, as it is used at each time-step of the computation.

Following the algorithm presented in the previous section, but based on the initial guess for the pressure given by Eq. (27), the estimated value for the equilibrium mass fraction $Y_{g}^{*}$ is seen in Fig. 5. b to be considerably improved, down to an error of about $0.5 \%$. The resulting final algorithm is summarized hereafter. 


\section{Summary of the procedure:}

Input : $p, \rho, T, Y_{l}$ or $Y_{g}$ from the hyperbolic step.

Output: $Y_{l}^{*}$ or $Y_{g}^{*}$ the equilibrium mass fractions.

\section{1) Search of pure fluid thermodynamic conditions}

- Limit cases $Y_{l}^{*} \rightarrow \epsilon$ and $Y_{g}^{*} \rightarrow \epsilon$ are considered with computation of the pressure and temperature according to the mixture equation of state (8) and (10).

- If either subcooled liquid or superheated thermodynamic conditions are found according to Eq. (18), then $Y_{l}^{*}$ or alternately $Y_{g}^{*}$ is either $\epsilon$ or $1-\epsilon$.

2) Otherwise, the thermodynamic equilibrium must be found

a) Estimation of the equilibrium pressure

- Calculation of $p_{1}^{*}$ according to Eq. (25) using the temperature from the hyperbolic step.

- Calculation of $p_{2}^{*}$ according to Eq. (26) using the temperature and pressure from the hyperbolic step.

- Prediction of the equilibrium pressure $p^{*}$ according to Eq. (27) using Eqs. (44), (21), (22) and (28). The mixture internal energy and specific volume come from the hyperbolic step.

\section{b) Equilibrium computation}

- Computation of the liquid mass fraction at equilibrium $Y_{l}^{*}$ according to the Minmod-like procedure (Eqs. (23) and (24)) presented in Sec. 3 using Eqs. (4), (21) and (22) estimated at $p^{*}$ and $T^{*}=T_{\text {sat }}\left(p^{*}\right)$.

\section{Results}

In order to illustrate the robustness and the accuracy of the relaxation algorithm, a shock tube containing liquid water and its own vapor is considered, with variable initial conditions. Shock tube tests appear as excellent benchmarks as the flow contains shock waves, contact discontinuities and rarefaction fans that create some arduous conditions. In this section, the tube is 1 meter long and the initial discontinuity is located at 0.5 meter. Boundary conditions are considered as non-reflecting. The computations that follow are addressed with the first-order Godunov method and the HLLC Riemann solver (see Toro (1997) [24], Saurel et al. (2016) [13], for details). Obviously, higher order extensions can be considered, but this is not the scope of the present work.

The initial conditions correspond to both saturated liquid and vapor each time a mixture 
is present initially. Given initial pressures and mass fractions, the initial temperatures are computed with Eq. (5), initial mixture energy and specific volumes are deduced from the definitions given by Eqs. (2).

This section presents the results obtained with the present relaxation solver and a classic root-finding procedure method such as Newton's.

\subsection{Shock tube test with a mixture containing mainly water vapor}

A two-phase mixture with an initial vapor mass fraction of 0.8 is considered throughout the entire tube with an initial pressure ratio of 2 , resulting in the presence of initial density and temperature discontinuities. The results are shown at time $t \approx 0.8 \mathrm{~ms}$ in Fig. 8 ,
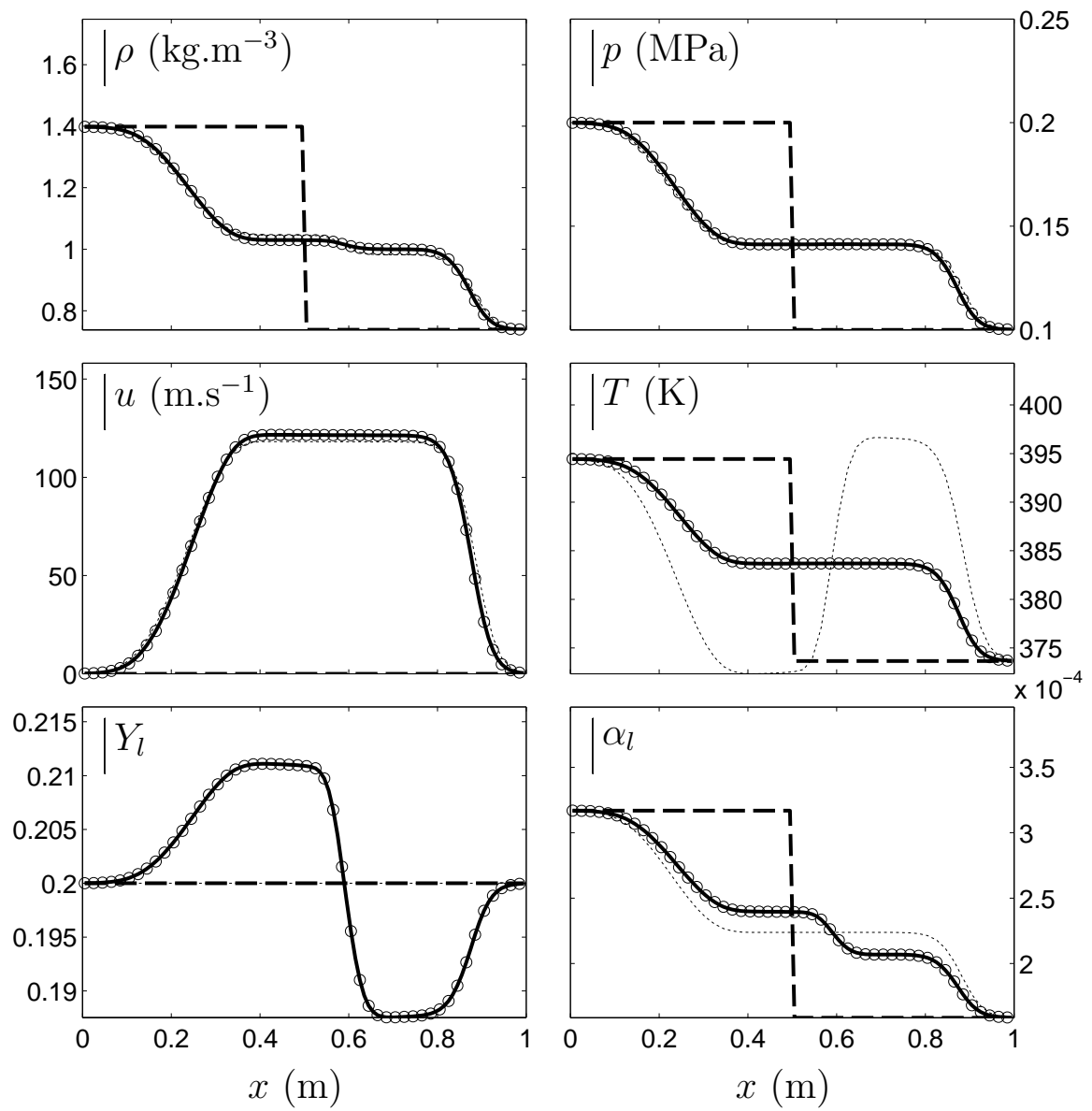

Figure 8: Comparison of the present relaxation algorithm (thick lines) versus the non-linear Newton's method (symbols). Shock tube test with a two-phase mixture mainly made of water vapor. The dotted lines represent the solutions without phase transition. The dashed lines represent the initial conditions: $p_{l}=2 \cdot 10^{5} \mathrm{~Pa}, p_{r}=10^{5} \mathrm{~Pa}$, $u_{l}=u_{r}=0 \mathrm{~m} . \mathrm{s}^{-1}, Y_{l}^{l i q}=Y_{r}^{l i q}=0.2$. Final time: $t \approx 0.8 \mathrm{~ms}$. Mesh: 100 cells. For the sake of visualization, only 50 symbols out of 100 are plotted for the non-linear Newton's method. 
Perfect agreement is obtained between the present relaxation solver and the non-linear iterative method recalled in Appendix. Clearly the shock compression yields evaporation and the rarefaction results into condensation. Note that the initial left and right sides are now connected with the same temperature in the central area of the tube. This temperature being the saturation temperature at the current pressure $T^{*}=T_{\text {sat }}\left(p^{*}\right)$. Obviously without phase transition, two different temperatures evolve at the central region, separated by a contact wave.

\subsection{Shock tube test with a vanishing liquid phase}

We now address a similar test as the previous one but with a vanishing phase. The initial liquid mass fraction is 0.01 throughout the entire tube. The results are shown at time $t \approx 0.5 \mathrm{~ms}$ in Fig. 9,

Perfect agreement between the two solvers is again obtained. Here the shock compression of the liquid-gas mixture yields total evaporation. Oppositely, the expansion wave yields condensation. Appearance of pure gas is computed without oscillations by the two methods.

\subsection{Shock tube test with a mixture containing mainly liquid water}

A two-phase flow with an initial liquid mass fraction of 0.8 throughout the entire tube is now considered with the same pressure ratio as previously. The results are shown at time $t \approx 1.5 \mathrm{~ms}$ in Fig. 10.

A condensation process is now observed at the shock while evaporation (cavitation) appears through the rarefaction fan. The agreement between the two methods is again excellent.

\subsection{Shock tube test with a vanishing gas phase}

The behavior of the same methods is now examined with a vanishing vapor phase. The same two-phase flow shock tube is addressed with an initial liquid mass fraction of 0.999 throughout the entire tube. The results are shown at time $t \approx 8 \mathrm{~ms}$ in Fig. 11.

Condensation again appears towards the shock and evaporation through expansion waves. Perfect agreement between the two methods is again observed. Pure liquid appearance is obtained without oscillations. 

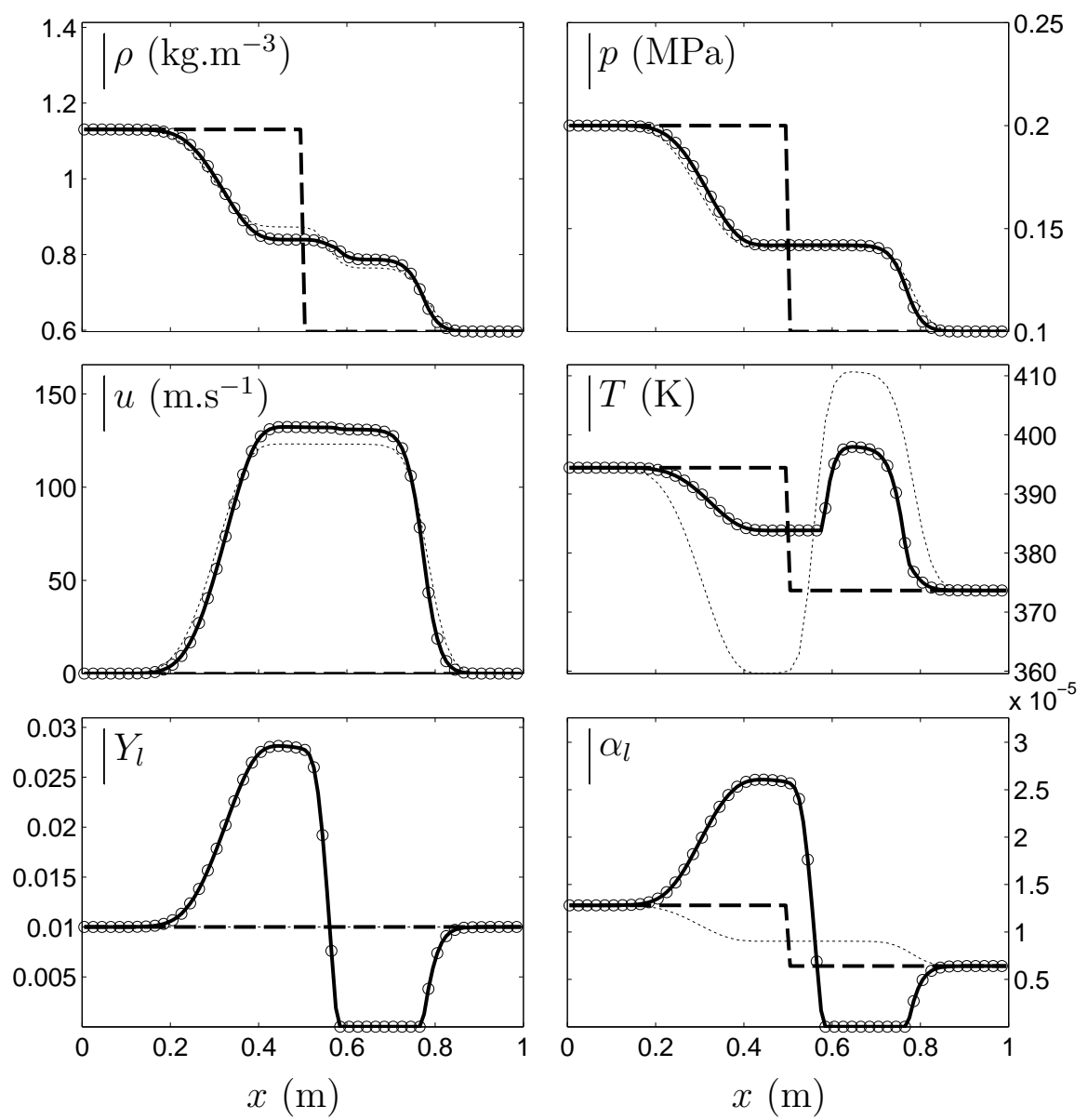

Figure 9: Comparison of the present relaxation algorithm (thick lines) versus the non-linear Newton's method (symbols). Shock tube test with a two-phase mixture involving a vanishing liquid phase. The dotted lines represent the solutions without phase transition. The dashed lines represent the initial conditions: $p_{l}=2 \cdot 10^{5}$ $\mathrm{Pa}, p_{r}=10^{5} \mathrm{~Pa}, u_{l}=u_{r}=0 \mathrm{~m} . \mathrm{s}^{-1}, Y_{l}^{l i q}=Y_{r}^{l i q}=0.01$. Final time: $t \approx 0.5 \mathrm{~ms}$. Mesh: 100 cells. For the sake of visualization, only 50 symbols out of 100 are plotted for the non-linear Newton's method. 

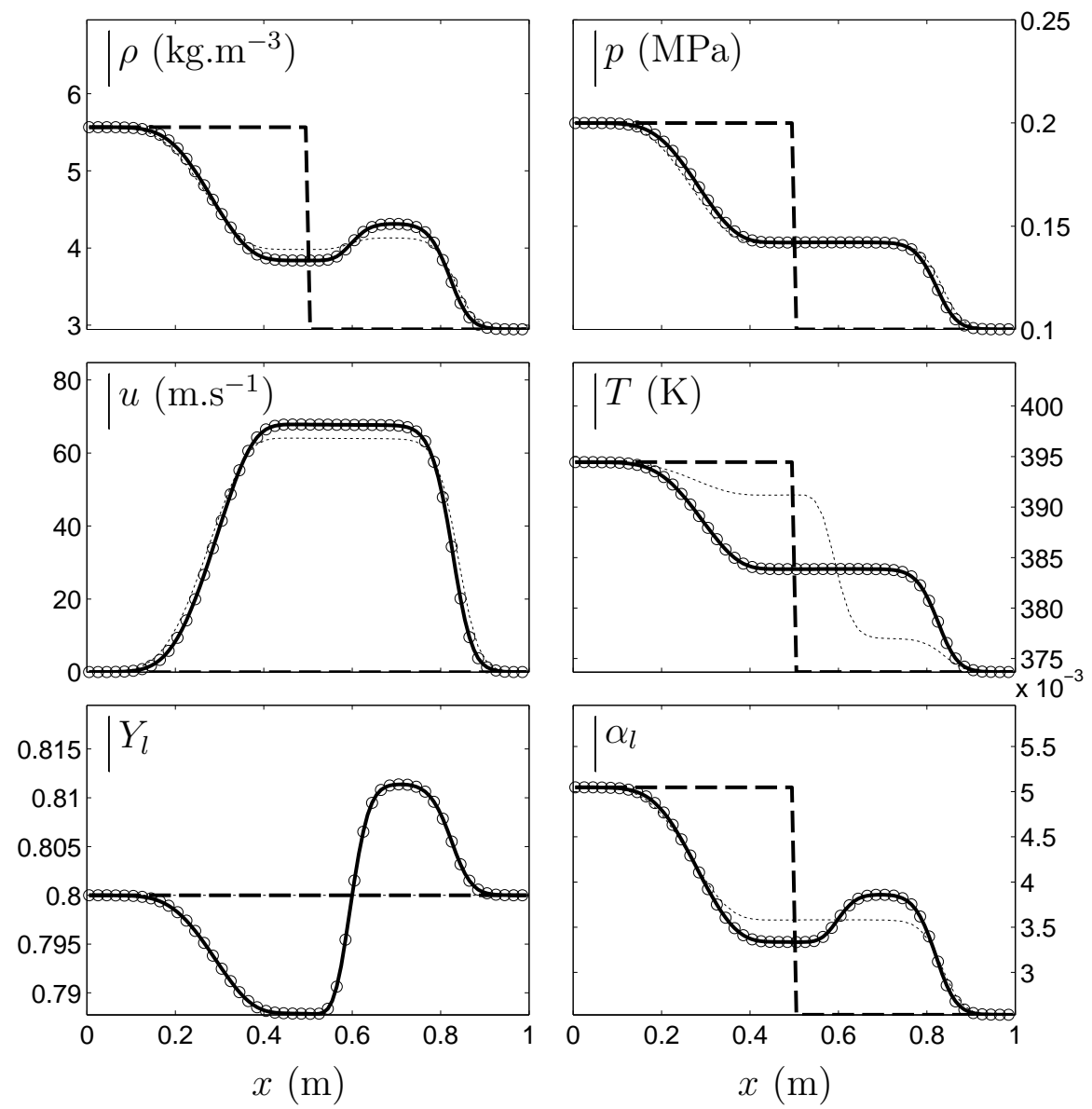

Figure 10: Comparison of the present relaxation algorithm (thick lines) versus the non-linear Newton's method (symbols). Shock tube test with a two-phase mixture mainly composed of liquid water. The dotted lines represent the solutions without phase transition. The dashed lines represent the initial conditions: $p_{l}=2 \cdot 10^{5}$ $\mathrm{Pa}, p_{r}=10^{5} \mathrm{~Pa}, u_{l}=u_{r}=0 \mathrm{~m} . \mathrm{s}^{-1}, Y_{l}^{\text {liq }}=Y_{r}^{\text {liq }}=0.8$. Final time: $t \approx 1.5 \mathrm{~ms}$. Mesh: 100 cells. For the sake of visualization, only 50 symbols out of 100 are plotted for the non-linear Newton's method. 

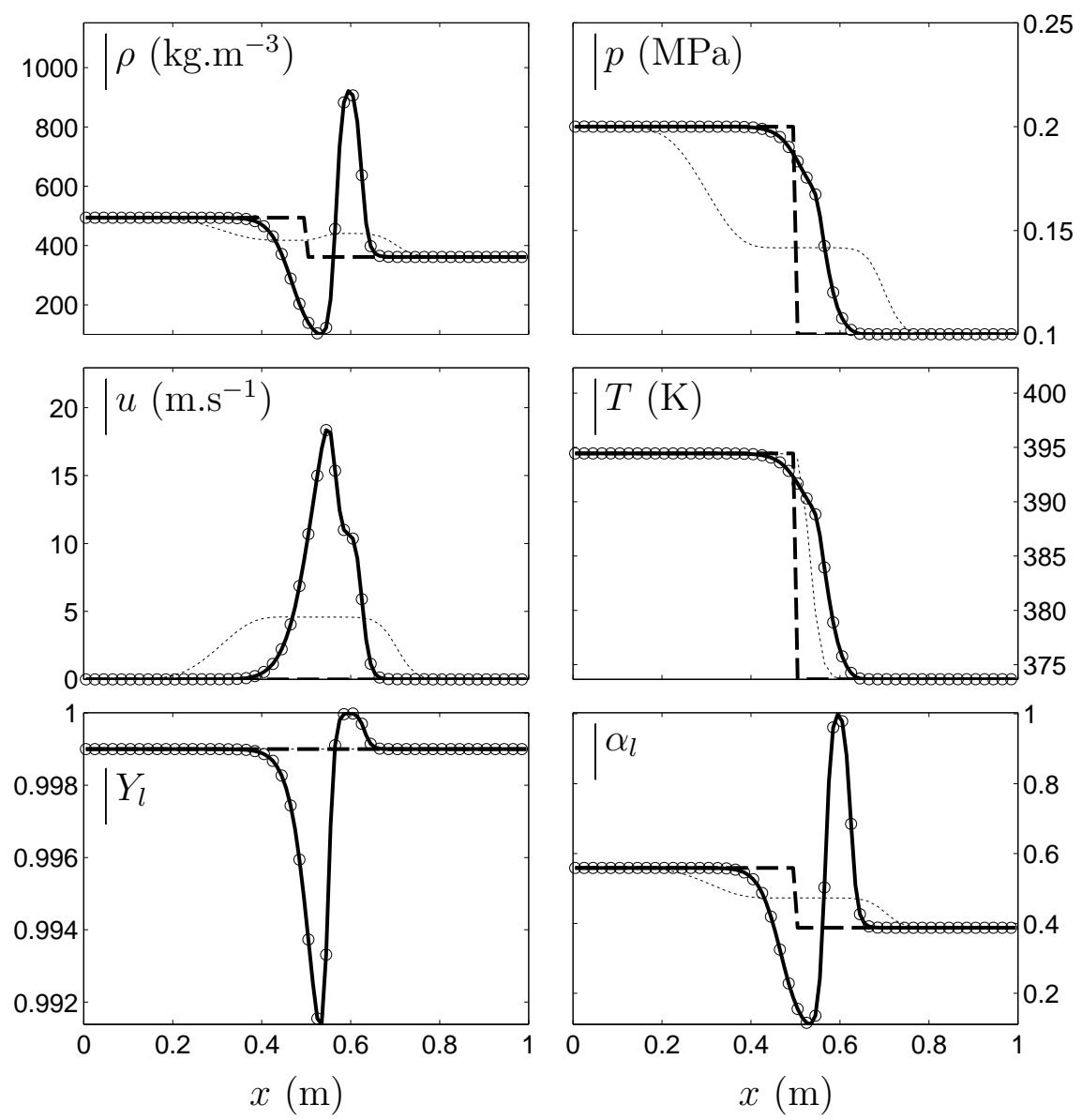

Figure 11: Comparison of the present relaxation algorithm (thick lines) versus the non-linear Newton's method (symbols). Shock tube test with a two-phase mixture involving a vanishing vapor phase. The dotted lines represent the solutions without phase transition. The dashed lines represent the initial conditions: $p_{l}=2 \cdot 10^{5}$ $\mathrm{Pa}, p_{r}=10^{5} \mathrm{~Pa}, u_{l}=u_{r}=0 \mathrm{~m} . \mathrm{s}^{-1}, Y_{l}^{l i q}=Y_{r}^{l i q}=0.999$. Final time: $t \approx 8 \mathrm{~ms}$. Mesh: 100 cells. For the sake of visualization, only 50 symbols out of 100 are plotted for the non-linear Newton's method. 


\subsection{Superheated vapor}

Considering pure fluids at initial states, it is interesting to set initial conditions away from the thermodynamic equilibrium. Superheated pure vapor is considered throughout the tube. The temperature is therefore above the saturation temperature and is set at $450 \mathrm{~K}$. The pressure is set at $10^{5} \mathrm{~Pa}$. The initial velocity is set at $-200 \mathrm{~m} \cdot \mathrm{s}^{-1}$ at left and $+200 \mathrm{~m} \cdot \mathrm{s}^{-1}$ at right. The results are given at time of $t \approx 0.3 \mathrm{~ms}$ in Fig. 12. The vapor expends and gets colder but remains vapor, as expected.
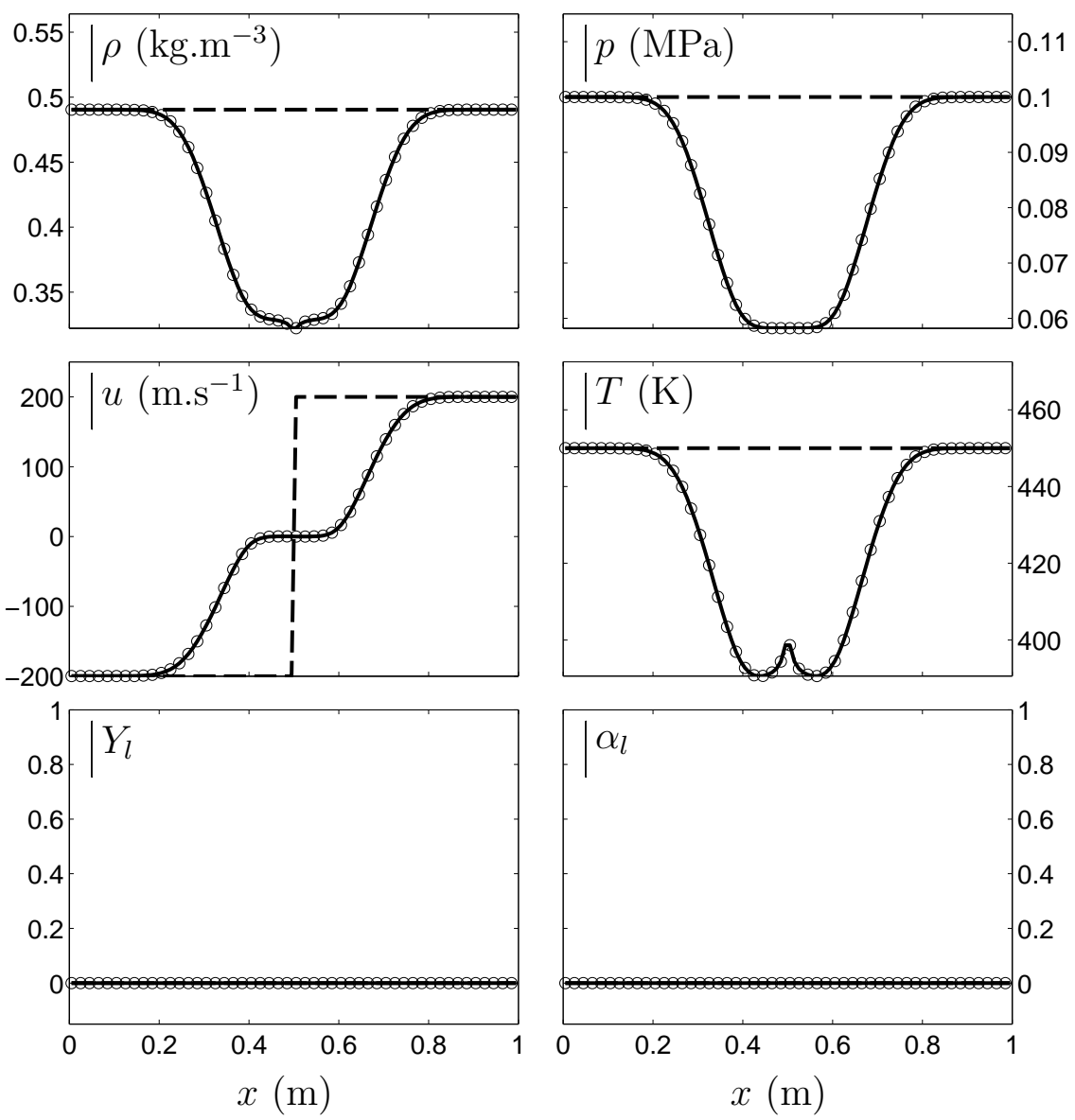

Figure 12: Comparison of the present relaxation algorithm (thick lines) versus the non-linear Newton's method (symbols). Double expansion test with superheated vapor. The dotted lines represent the solutions without phase transition. The dashed lines represent the initial conditions: $p_{l}=10^{5} \mathrm{~Pa}, p_{r}=10^{5} \mathrm{~Pa}, u_{l}=-200 \mathrm{~m} . \mathrm{s}^{-1}, u_{r}=$ $200 \mathrm{~m} . \mathrm{s}^{-1}, Y_{l}^{l i q}=Y_{r}^{l i q} \approx 0$. Final time: $t \approx 0.3 \mathrm{~ms}$. Mesh: 100 cells. For the sake of visualization, only 50 symbols out of 100 are plotted for the non-linear Newton's method. The vapor expands but remains vapor. 


\subsection{Subcooled liquid}

Let us now mirror the previous test and consider a pure liquid initially at $350 \mathrm{~K}$ corresponding to a subcooled liquid. The pressure is still initially set at $10^{5} \mathrm{~Pa}$ and the initial velocities are unchanged (double expansion). The results are given at two different times, $t \approx 0.165 \mathrm{~ms}$ in Fig. 13 and $t \approx 0.65 \mathrm{~ms}$ in Fig. 14, exhibiting an interesting behavior.
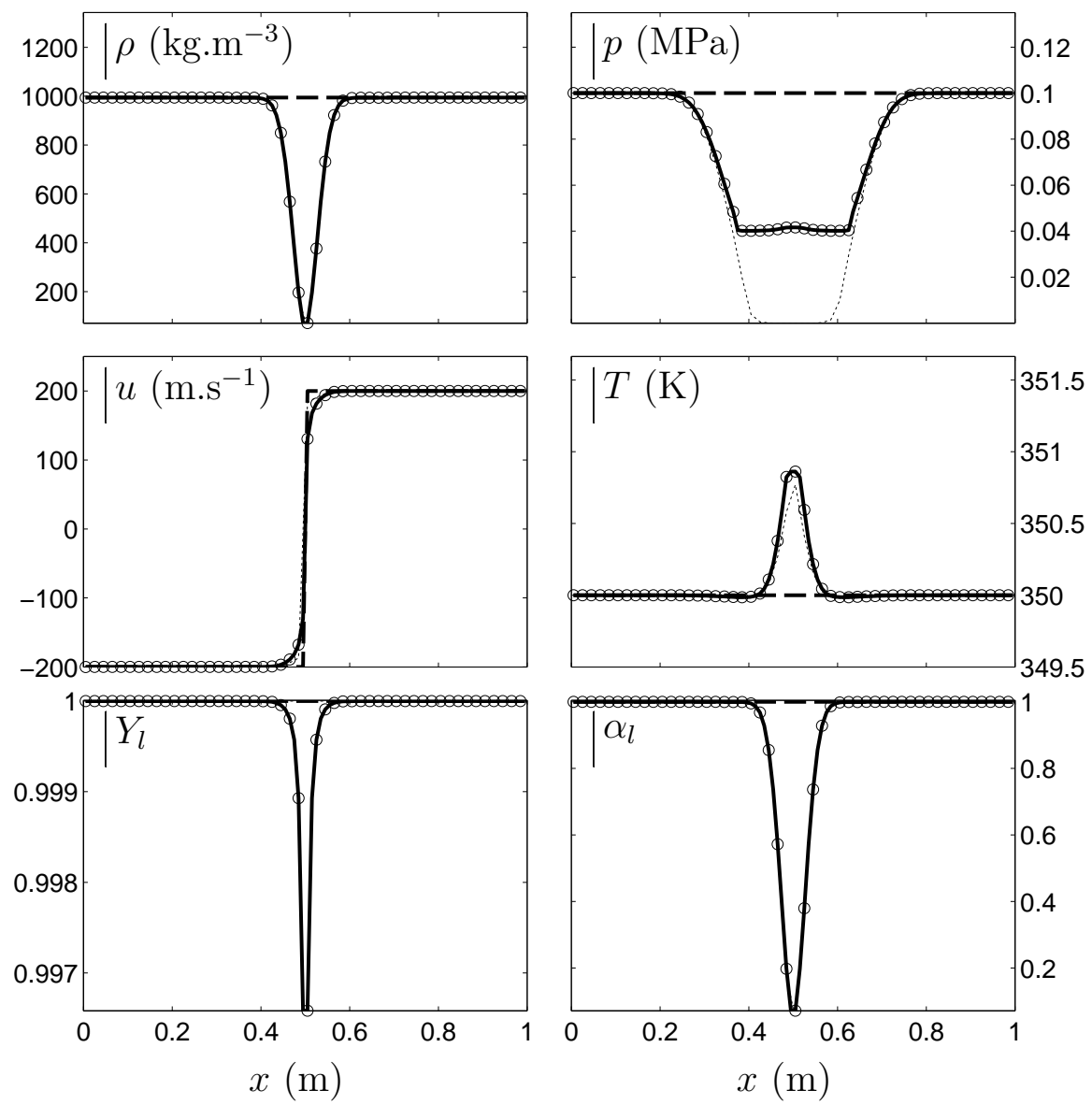

Figure 13: Comparison of the present relaxation algorithm (thick lines) versus the non-linear Newton's method (symbols). Double expansion test with subcooled liquid. Results are shown at early times $(t \approx 0.165 \mathrm{~ms})$ showing two fast expansion waves emitted into the pure liquid, and propagating at the liquid sound speed $\left(u \pm c_{l i q}\right)$. The next Figure shows the evolution at longer times, where two additional waves (evaporation fronts) are emitted. The dotted lines represent the solutions without phase transition. The dashed lines represent the initial conditions: $p_{l}=10^{5} \mathrm{~Pa}, p_{r}=10^{5} \mathrm{~Pa}, u_{l}=-200 \mathrm{~m} . \mathrm{s}^{-1}, u_{r}=200 \mathrm{~m} . \mathrm{s}^{-1}, Y_{l}^{l i q}=Y_{r}^{l i q} \approx 1$. Mesh: 100 cells. For the sake of visualization, only 50 symbols out of 100 are plotted for the non-linear Newton's method.

In Fig. 13 fast expansion waves are emitted and propagate into the pure liquid at the sound speed of this phase. They induce pressure drop from $0.1 \mathrm{MPa}$ to $0.04 \mathrm{MPa}$, corresponding to the saturation pressure at the current temperature. This temperature is shown in the same 

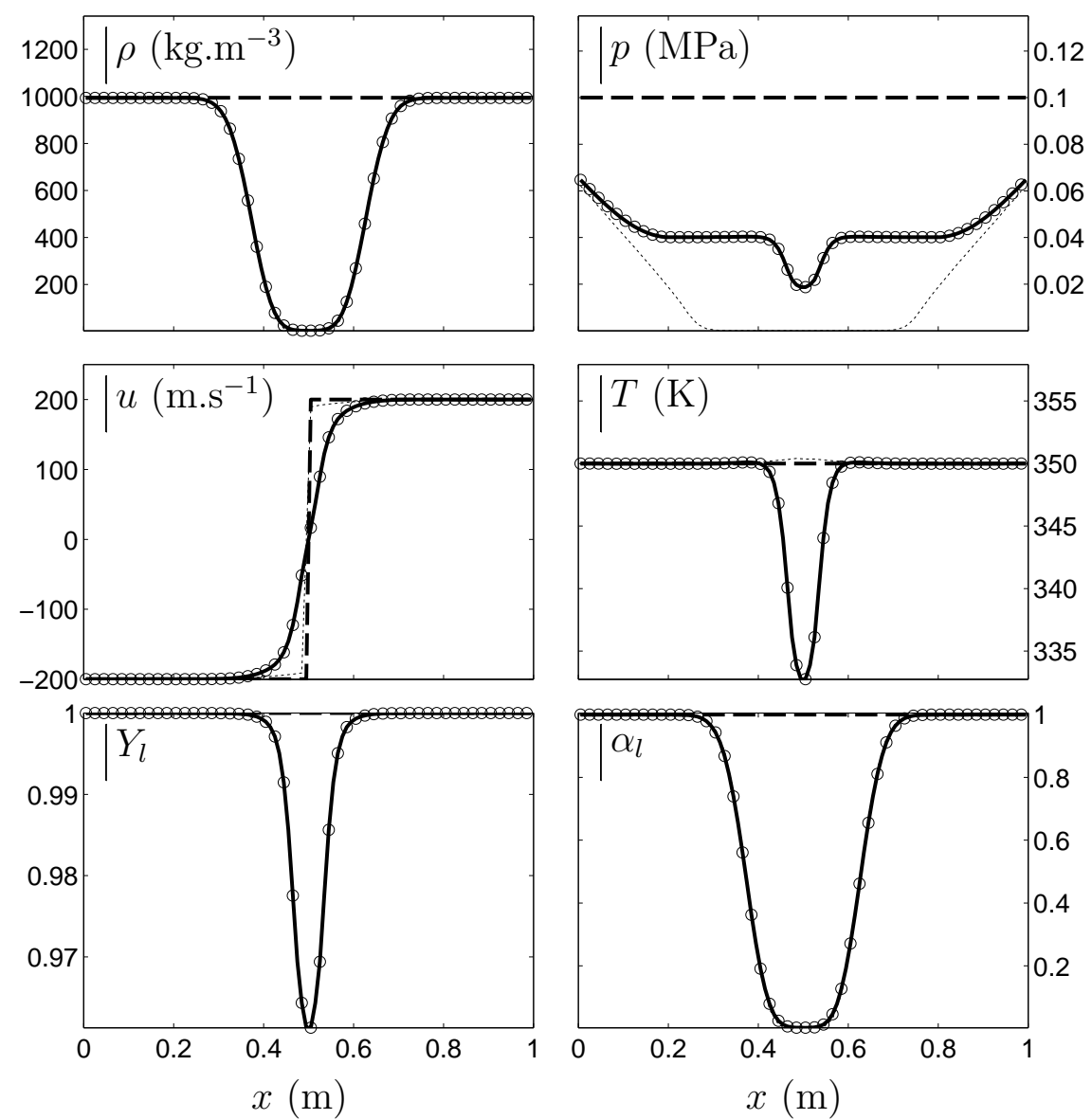

Figure 14: Evolution of the test case of Fig. 13 at longer times (here $t \approx 0.65 \mathrm{~ms}$ ). In addition to the first two expansion waves propagating with the liquid sound speed $\left(u \pm c_{l i q}\right)$; two evaporation fronts appear, propagating with the liquid/gas mixture sound speed at equilibrium $\left(u \pm c_{e q}\right)$. 
figure and is nearly constant in the domain, except at the center. This unphysical (but small) temperature increase is well known with all conservative methods and is related to the kinetic energy computation, that is badly computed from the momentum equation (see for example Cocchi et al. (1998) [25]). Due to this slight temperature increase and pressure drop, a slight variation of the liquid mass fraction appears.

From the expanded liquid at saturation pressure (0.04MPa) expansion continues as the right and left states still have expansion motion. Two new expansion waves now appear and are clearly visible in Fig. 14. They induce a pressure jump from 0.04MPa to 0.02MPa. These waves are evaporation fronts (Saurel et al. (2008) [6]) that propagate at $u \pm c_{e q}, c_{e q}$ being the mixture sound speed at equilibrium, lower than the sound speed given by Eq. (12). Now evaporation is significant, as about $4 \%$ of the liquid mass evaporates.

From these initial conditions of subcooled liquid in expansion, five different waves are emitted as shown in Fig. 15,

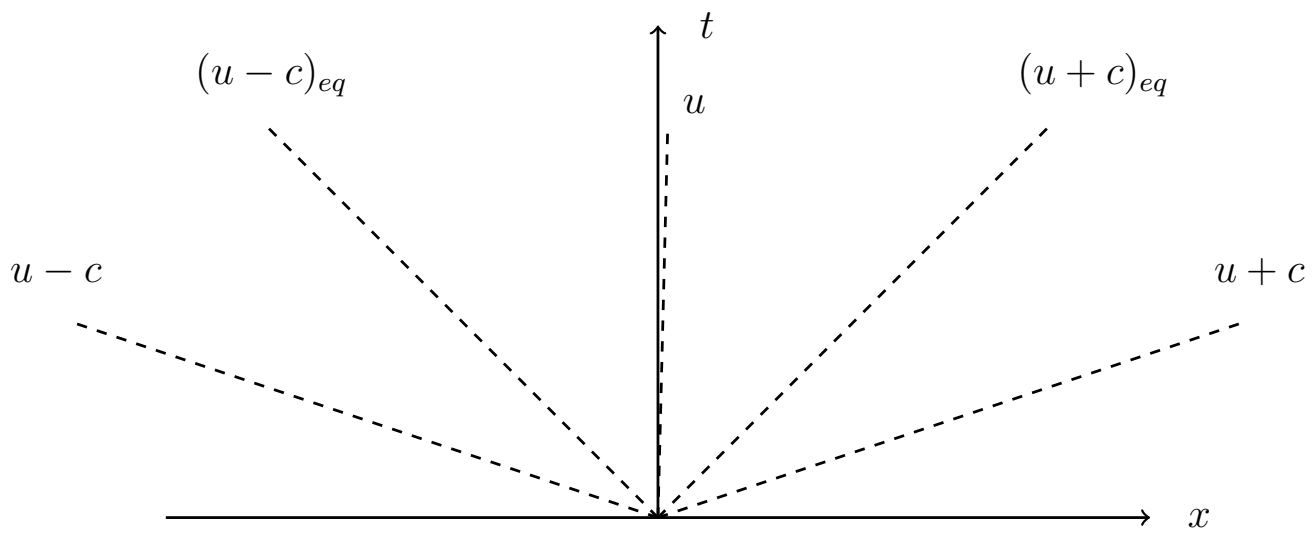

Figure 15: Schematic representation in a $(\mathrm{x}, \mathrm{t})$ diagram of the five waves present in Figs. 13 and 14,

Four of these waves are clearly visible in Fig. 13 and Fig. 14 as they correspond to the expansion waves already discussed. The contact wave propagating at the velocity $u=0 \mathrm{~m} \cdot \mathrm{s}^{-1}$ is not visible for symmetry reasons. These results confirm the observations of Saurel et al. (2008) [6] regarding evaporation front dynamics in flashing flows. They also explain the kinetic relation of Chaves (1984) [26] which considers evaporation fronts as CJ deflagration waves. They just correspond to expansion waves of the equilibrium model. 


\section{Computational time, efficiency and simplicity}

In most computational examples considered previously the computational times of the relaxation solver and the non-linear Newton's method are comparable. When dealing with test problems where a pure (or nearly pure) liquid is present, the relaxation algorithm is much faster. For example, Table 2 displays the different runtime gains for the tests shown in Figs 8 and 13 .

\begin{tabular}{|l|c|c|}
\hline Test & $\frac{\text { Relaxation solver runtime }}{\text { Newton's method untime }}$ & Runtime gain \\
\hline Fig 8 & 0.95 & $5 \%$ \\
Fig 13 & 0.50 & $50 \%$ \\
\hline
\end{tabular}

Table 2: Comparison of the average computational time and gain for the tests shown in Figs 8 and 13, Meshes of 500, 1000, 1500 and 2000 cells were used and yielded the same conclusions.

The main argument with the present method is consequently more related to its simplicity: the relaxation solver presents a straight-forward implementation whereas an iterative method requires solving a non-linear-algebraic model that may cause difficulties as a result of nonlinearities and single phase bounds of pure liquid and pure vapor, where it becomes ill posed. Besides, the root-finding Newton's method requires the calculation of the saturation pressure via Eq. (5) at each iterative step, which itself requires an iterative method.

The reliability of the present method is quite independent of the mesh resolution or the order of accuracy of the numerical scheme. For instance, Fig [16 repeats the test case already presented in Fig 9, with a 10-cell mesh and first order Godunov numerical scheme. These results are very reasonable, proving robustness of the method. Results for the same test case are also given in Fig 16 for a higher-order solver (MUSCL-Hancock scheme with the Superbee limiter), on a 1000-cell mesh. Again, perfect agreement is found between the present relaxation solver and the non-linear Newton's method.

Moreover, the present method easily extends to more than one gas constituent, for example when a non-condensable gas is present in addition to the vapor. Such extension is possible with the iterative Newton's method, but difficult. 

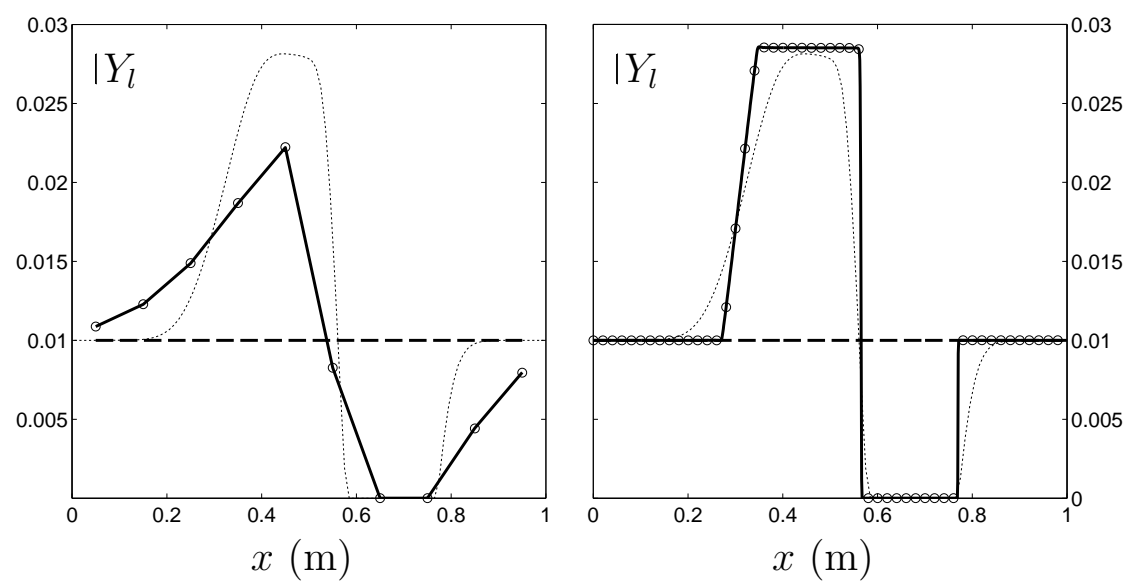

Figure 16: Comparison of the present relaxation algorithm (thick lines) versus the non-linear Newton's method (symbols). Shock tube test case with a two-phase mixture involving a vanishing liquid phase, computed on coarse (left figure) and fine (right figure) meshes for assessing method robustness. The dashed lines represent the initial conditions: $p_{l}=2 \cdot 10^{5} \mathrm{~Pa}, p_{r}=10^{5} \mathrm{~Pa}, u_{l}=u_{r}=0 \mathrm{~m} . \mathrm{s}^{-1}, Y_{l}^{l i q}=Y_{r}^{l i q}=0.01$. Final time: $t \approx 0.5 \mathrm{~ms}$. The figure on the left represents the solutions with a 10-cell mesh and first order Godunov numerical scheme. The figure on the right represents the solutions with a 1000-cell mesh and MUSCL-Hancock scheme with Superbee limiter. Only 50 symbols out of 1000 are plotted for the non-linear Newton's method in the figure on the right. The dotted lines represent the solution given in Fig 9 with a 100-cell mesh and first order Godunov numerical scheme. The relaxation method and iterative one tend to the same solution even with the coarse mesh.

\section{Multi-dimensional illustrations}

In this section, the capabilities of the flow model are illustrated on an evaporating liquid jet configuration, reminiscent of coaxial liquid jets, in conditions typical of cryotechnic rocket engines at ignition. The flow contains liquid oxygen and vapor oxygen at cryogenic temperatures. The stiffened gas parameters for oxygen are given in Table 3 .

\begin{tabular}{|l|c|c|}
\hline Coefficients & Liquid phase & Vapor phase \\
\hline$C_{p}(\mathrm{~J} / \mathrm{kg} / \mathrm{K})$ & 1702 & 780 \\
\hline$C_{v}(\mathrm{~J} / \mathrm{kg} / \mathrm{K})$ & 695 & 531 \\
\hline$\gamma$ & 2.45 & 1.47 \\
\hline$P_{\infty}(\mathrm{bar})$ & 1062 & 0 \\
\hline$q(\mathrm{~J} / \mathrm{kg})$ & -258000 & 6900 \\
\hline$q^{\prime}(\mathrm{J} / \mathrm{kg} / \mathrm{K})$ & 0 & -9280 \\
\hline
\end{tabular}

Table 3: Stiffened gas coefficients for oxygen.

The 2D computations are carried out on a simplified geometry given in Fig. 17.

The inlet boundary conditions correspond to two subsonic inflows: 


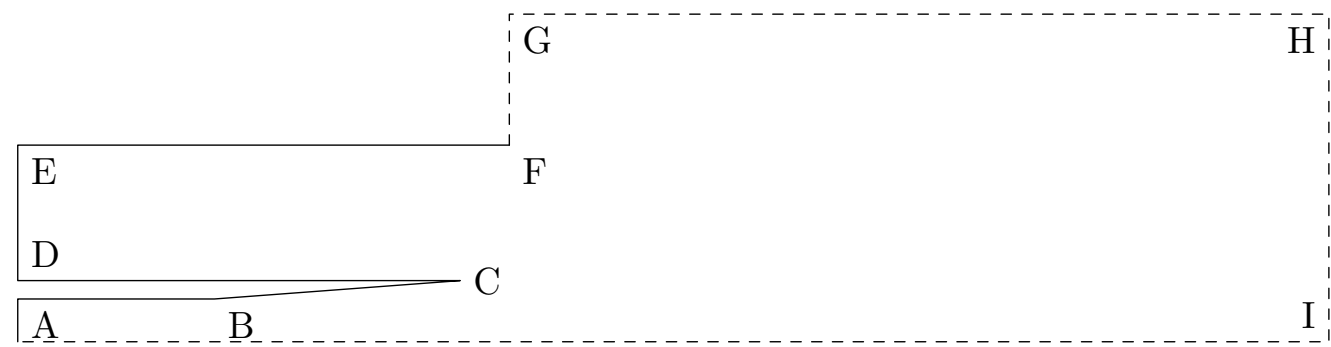

\begin{tabular}{|c|c|c||c|c|c|}
\hline & $\mathrm{X}$ (abscissa) $(\mathrm{mm})$ & $\mathrm{Y}(\mathrm{mm})$ & & $\mathrm{X}($ abscissa $)(\mathrm{mm})$ & $\mathrm{Y}(\mathrm{mm})$ \\
\hline $\mathrm{A}$ & -20 & 1.75 & $\mathrm{E}$ & -20 & 8 \\
\hline B & -12 & 1.75 & $\mathrm{~F}$ & 0 & 8 \\
\hline C & -2 & 2.5 & $\mathrm{G}$ & 0 & 40 \\
\hline $\mathrm{D}$ & -20 & 2.5 & $\mathrm{H}$ & 100 & 40 \\
\hline
\end{tabular}

Figure 17: Geometrical data for half the computational domain.

- central flow made of pure liquid oxygen, at $100 \mathrm{~K}$ and $30 \mathrm{~m} . \mathrm{s}^{-1}$, pressure $1 \mathrm{MPa}$ along segment AB of Fig. 17,

- peripheral flow made of pure gaseous oxygen, at $150 \mathrm{~K}$ and $200 \mathrm{~m} . \mathrm{s}^{-1}$, with the pressure of $1 \mathrm{MPa}$ along segment DE of Fig. 17.

At the outlet, along segment IH, a non-reflecting subsonic boundary condition is imposed at $1 \mathrm{MPa}$. The remaining walls are treated as symmetric boundary conditions. The mesh consists of 360000 triangles, with a spatial discretization varying from $0.1 \mathrm{~mm}$ in the jet to $0.6 \mathrm{~mm}$ in the far field.

The small scale destabilization of the liquid jet requires to extend the numerical solver presented in Saurel et al. (2016) [13] to second order. This was achieved following the MUSCLHancock scheme with the Superbee limiter, as detailed for exemple in Toro (1997) [24].

Two computations have been carried out on the same mesh, with the aim of studying the effect of the thermochemical relaxation on the jet destabilization. The contours of mixture density as obtained with and without evaporation effects are compared in Fig. 18,

The filaments separating the main liquid core and the gas gradually vanish as a consequence of evaporation. Fig [19] compares the corresponding temperature contours. The thermochemical relaxation solver has the expected behavior: pure liquid oxygen and pure gas oxygen are free to 


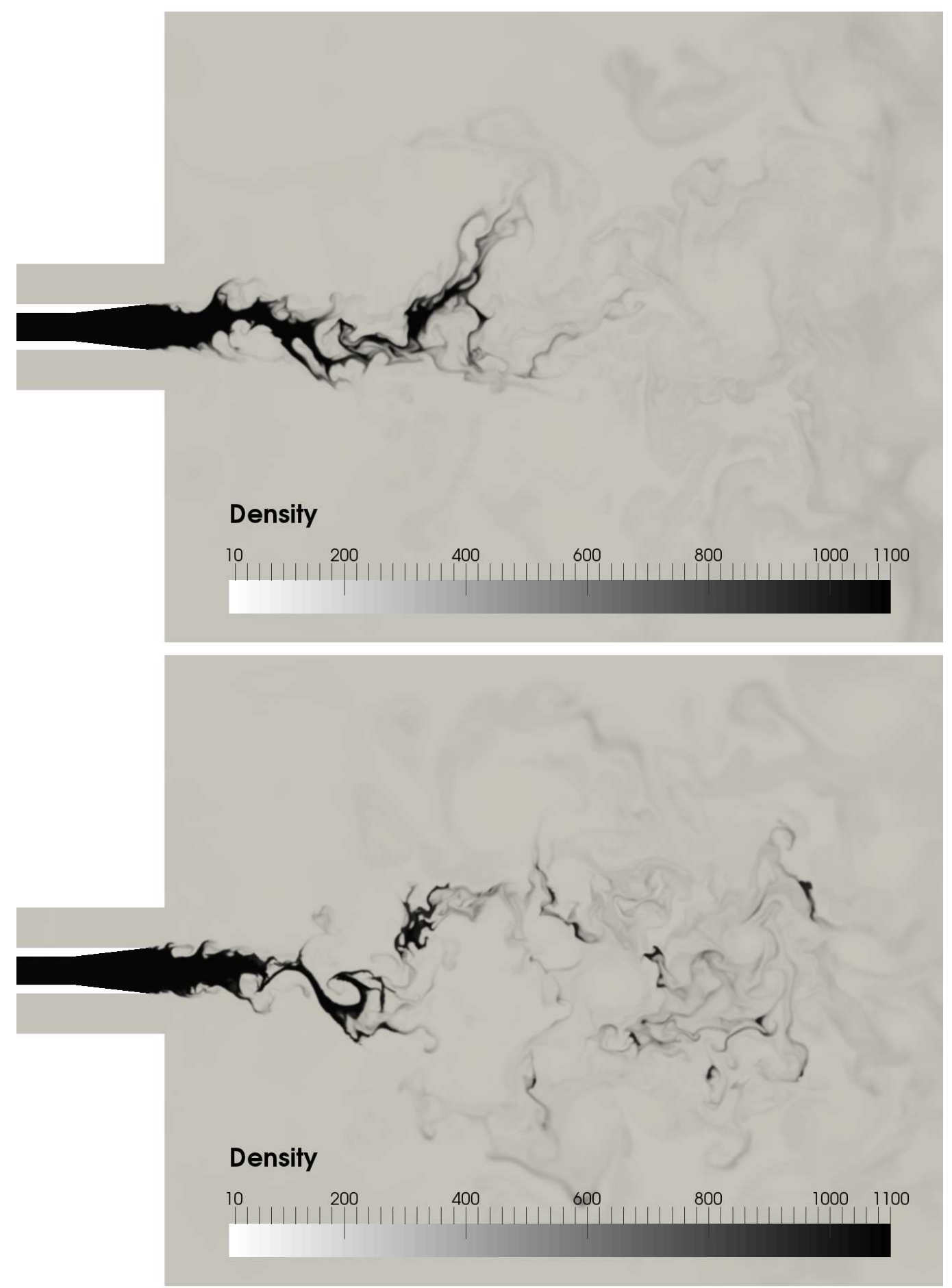

Figure 18: Mixture density without (top view) and with (bottom view) evaporation. 

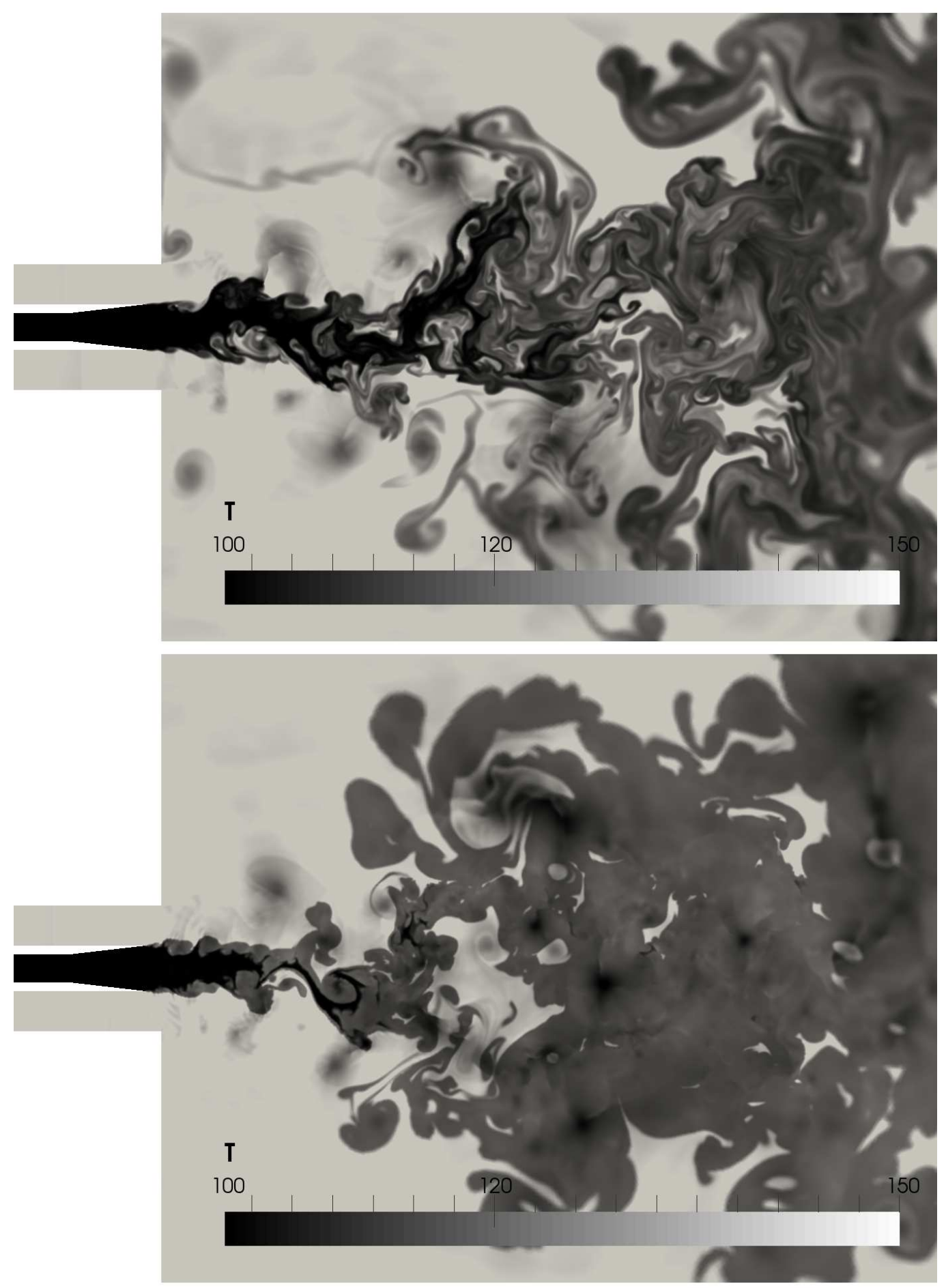

Figure 19: Temperature without (top view) and with (bottom view) evaporation. 
evolve out of the saturation conditions (resp. at $100 \mathrm{~K}$ and $150 \mathrm{~K}$ ), whereas $T=T_{\text {sat }}(p)$ where the flow consists of a two-phase mixture.

For the configuration tested here, the CPU saving of the present algorithm over the iterative method is of the order of $18 \%$, with no noticeable change in the results.

In future works, the thermochemical relaxation solver will be extended to take into account a multicomponent gas mixture $\left(\mathrm{H}_{2}, \mathrm{He}, \mathrm{H}_{2} \mathrm{O}\right.$, etc. $)$ which is present in many applications. Also, capillary effects are not included in these simulations, as the intense velocity gradients make them negligible in the present jet destabilization conditions. However, capillary effects may be of importance when the filaments start separating from the jet, and our future work will include them, following the strategy presented in Le Martelot et al. (2014) [14].

\section{Conclusions}

A simple relaxation solver has been built to compute phase equilibrium in multiphase compressible flows. Computational examples have shown that the method converges to the same solution as methods based on iterative (and exact) equilibrium solvers. The new method uses two main ingredients to estimate the liquid (or vapor) equilibrium mass fraction:

- Minmod-type treatment of the source term.

- An accurate estimate of the equilibrium pressure based on some linearization of the equilibrium mass fraction sources as functions of the pressure.

The method also considers single phase limits made of pure liquid and pure vapor. The next step will be to address gas mixtures in conjunction with a liquid phase, in the direction of two-phase combustion.

\section{Acknowledgements}

Part of this work has been carried out in the framework of the Labex MEC (ANR-10LABX-0092) and of the A*MIDEX project (ANR-11-IDEX-0001-02), funded by the Investissements d'Avenir French Government program managed by the French National Research Agency (ANR). We also acknowledge funding from ANR through project ANR-14-CE22-0014. 


\section{References}

[1] B. Abramzon, W. Sirignano, Droplet vaporization model for spray combustion calculations, International Journal of Heat and Mass Transfer 32 (9) (1989) 1605-1618.

[2] M. Jacobson, Fundamentals of atmospheric modeling, Cambridge University Press, 2005.

[3] D. Furfaro, R. Saurel, Modeling droplet phase change in the presence of a multi-component gas mixture, Applied Mathematics and Computation 272 (2016) 518-541.

[4] M. Baer, J. Nunziato, A two-phase mixture theory for the deflagration-to-detonation transition (DDT) in reactive granular materials, International Journal of Multiphase Flow 12 (6) (1986) 861-889.

[5] A. Kapila, R. Menikoff, J. Bdzil, S. Son, D. Stewart, Two-phase modeling of deflagrationto-detonation transition in granular materials: Reduced equations, Physics of Fluids 13 (10) (2001) 3002-3024.

[6] R. Saurel, F. Petitpas, R. Abgrall, Modelling phase transition in metastable liquids: application to cavitating and flashing flows, Journal of Fluid Mechanics 607 (2008) 313-350.

[7] S. LeMartelot, B. Nkonga, R. Saurel, Liquid and liquid-gas flows at all speeds, Journal of Computational Physics 255 (2013) 53-82.

[8] P. Downar-Zapolski, Z. Bilicki, L. Bolle, J. Franco, The non-equilibrium relaxation model for one-dimensional flashing liquid flow, International Journal of Multiphase Flow 22 (3) (1996) 473-483.

[9] M. Barret, E. Faucher, J. Hérard, Schemes to compute unsteady flashing flows, AIAA Journal 40 (5) (2002) 905-913.

[10] H. Lund, A hierarchy of relaxation models for two-phase flow, SIAM Journal on Applied Mathematics 72 (6) (2012) 1713-1741. 
[11] A. Singhal, M. Athavale, H. Li, Y. Jiang, Mathematical basis and validation of the full cavitation model, Journal of Fluids Engineering 124 (3) (2002) 617-624.

[12] F. Petitpas, J. Massoni, R. Saurel, E. Lapebie, L. Munier, Diffuse interface model for high speed cavitating underwater systems, International Journal of Multiphase Flow 35 (8) (2009) 747-759.

[13] R. Saurel, P. Boivin, O. Le Métayer, A general formulation for cavitating, boiling and evaporating flows, Computers and Fluids 128 (2016) 53-64.

[14] S. Le Martelot, R. Saurel, B. Nkonga, Towards the direct numerical simulation of nucleate boiling flows, International Journal of Multiphase Flow 66 (2014) 62-78.

[15] H. Orbey, S. Sandler, Modeling vapor-liquid equilibria: Cubic equations of state and their mixing rules, Vol. 1, Cambridge University Press, 1998.

[16] G. Allaire, G. Faccanoni, S. Kokh, A strictly hyperbolic equilibrium phase transition model, Comptes Rendus Mathematique 344 (2) (2007) 135-140.

[17] G. Faccanoni, S. Kokh, G. Allaire, Modelling and simulation of liquid-vapor phase transition in compressible flows based on thermodynamical equilibrium, ESAIM : Mathematical Modelling and Numerical Analysis 46 (05) (2012) 1029-1054.

[18] O. Le Métayer, J. Massoni, R. Saurel, Dynamic relaxation processes in compressible multiphase flows. Application to evaporation phenomena, in: ESAIM: Proceedings, Vol. 40, EDP Sciences, 2013, pp. 103-123.

[19] B. Van Leer, Towards the ultimate conservative difference scheme. V. A second-order sequel to Godunov's method, Journal of Computational Physics 32 (1) (1979) 101-136.

[20] M. Lallemand, R. Saurel, Pressure relaxation procedures for multiphase compressible flows, INRIA Report 403 (8).

[21] O. Le Métayer, J. Massoni, R. Saurel, Elaborating equations of state of a liquid and its vapor for two-phase flow models; élaboration des lois d'état d'un liquide et de sa vapeur pour les 
modèles d'écoulements diphasiques, International Journal of Thermal Sciences 43 (3) (2004) 265-276.

[22] O. Le Métayer, R. Saurel, The Noble-Abel Stiffened-Gas equation of state, Physics of Fluids 28 (4) (2016) 046102.

[23] A. Wood, A textbook of sound, G. Bell and Sons Ltd., London, 1930.

[24] E. F. Toro, Riemann solvers and numerical methods for fluid dynamics: A practical introduction, Springer Science \& Business Media, 1997.

[25] J. Cocchi, R. Saurel, J. Loraud, Some remarks about the resolution of high velocity flows near low densities, Shock Waves 8 (2) (1998) 119-125.

[26] H. Chaves, Changes of phase and waves on depressurization of liquids with high specific heat, Mittl Maz Planck Institut Strmungsforschung 77.

\section{AppendixA. Analytical expressions for the iterative algorithm}

Based on the expressions of $Y_{l}^{m}\left(p^{*}\right)$ and $Y_{l}^{e}\left(p^{*}\right)$ from Eqs. (21) and (22), let us introduce the following function:

$$
f(p)=\left[e_{l}(p)-e_{g}(p)\right]\left[v-v_{g}(p)\right]-\left[e-e_{g}(p)\right]\left[v_{l}(p)-v_{g}(p)\right]
$$

whose solution is the exact equilibrium pressure obtained with Newton's iteration,

$$
p^{n+1}=p^{n}-\frac{f(p)}{f^{\prime}(p)} .
$$

The derivative of $f$ with respect to the pressure is given by,

$$
\left.\left.f^{\prime}(p)=\frac{d f}{d p}=\frac{\partial f}{\partial p}\right)_{T}+\frac{\partial f}{\partial T}\right)_{p} \frac{d T}{d p} .
$$

$\left.\frac{\partial f}{\partial p}\right)_{T}$ and $\left.\frac{\partial f}{\partial T}\right)_{p}$ are straightforward to derive analytically. In the case $p \ll p_{\infty, l}$, they reduce to,

$$
\left.\frac{\partial f}{\partial p}\right)_{T}=\frac{\left(\gamma_{g}-1\right) C_{v, g} T\left(\gamma_{l} C_{v, l} T-e+q_{l}\right)}{p^{2}}
$$




$$
\left.\frac{\partial f}{\partial T}\right)_{p}=\frac{C_{v, g}\left(-2 \gamma_{l}\left(\gamma_{g}-1\right) C_{v, l} T+\left(\gamma_{g}-1\right)\left(e-q_{l}\right)\right)+\left(\gamma_{l} C_{v, l}-C_{v, g}\right) p v}{p}
$$

To compute $\frac{d T}{d p}$ let us recall the saturation temperature.

$$
T=T_{s a t}(p)=-\frac{B}{C W\left(-\frac{B e^{A / C} p^{-1 / C}\left(p+p_{\infty, l}\right)^{D / C}}{C}\right)}
$$

where $\mathrm{W}$ is the Lambert $\mathrm{W}$ function. Differentiating the above function and making use of the Lambert $\mathrm{W}$ function leads to,

$$
\frac{d T_{s a t}}{d p}=\frac{B\left((D-1) p-p_{\infty, l}\right)}{C^{2} p\left(p+p_{\infty, l}\right) W\left(-\frac{B e^{A / C} p^{-1 / C}\left(p+p_{\infty, l}\right)^{D / C}}{C}\right)\left(W\left(-\frac{B e^{A / C} p^{-1 / C}\left(p+p_{\infty, l}\right)^{D / C}}{C}\right)+1\right)},
$$

which can be conveniently expressed as

$$
\frac{d T_{s a t}}{d p}=\frac{T_{s a t}^{2}\left((D-1) p-p_{\infty, l}\right)}{p\left(p+p_{\infty, l}\right)\left(B-C T_{s a t}\right)}
$$

